\title{
Critical Ising model in varying dimension by conformal bootstrap
}

\author{
Andrea Cappelli, ${ }^{a}$ Lorenzo Maffi ${ }^{a, b}$ and Satoshi Okuda ${ }^{c}$ \\ ${ }^{a}$ INFN - Sezione di Firenze, \\ Via G. Sansone 1, 50019 Sesto Fiorentino, Firenze, Italy \\ ${ }^{b}$ Dipartimento di Fisica, Università di Firenze, \\ Via G. Sansone 1, 50019 Sesto Fiorentino, Firenze, Italy \\ ${ }^{c}$ Department of Physics, Rikkyo University, \\ Toshima, Tokyo 171-8501, Japan \\ E-mail: andrea.cappelli@fi.infn.it, lorenzo.maffi@fi.infn.it, \\ sato.okuda@gmail.com
}

ABSTRACT: The single-correlator conformal bootstrap is solved numerically for several values of dimension $4>d>2$ using the available SDPB and Extremal Functional methods. Critical exponents and other conformal data of low-lying states are obtained over the entire range of dimensions with up to four-decimal precision and then compared with several existing results. The conformal dimensions of leading-twist fields are also determined up to high spin, and their $d$-dependence shows how the conformal states rearrange themselves around $d=2.2$ for matching the Virasoro conformal blocks in the $d=2$ limit. The decoupling of states at the Ising point is studied for $3>d>2$ and the vanishing of one structure constant at $d=3$ is found to persist till $d=2$ where it corresponds to a Virasoro null-vector condition.

KeYwords: Conformal and W Symmetry, Conformal Field Theory, Nonperturbative Effects

ARXiv EPrint: 1811.07751 


\section{Contents}

\section{Introduction 1}

2 Precise critical exponents and structure constants as functions of dimension

2.1 Method and $d=3$ checks 3

2.2 Polynomial fits of $4>d>2$ data and comparison with other approaches 6

$\begin{array}{lll}2.2 .1 & \text { Conformal dimensions } & 7\end{array}$

$\begin{array}{lll}2.2 .2 & \text { Structure constants } & 15\end{array}$

$\begin{array}{lll}2.3 & \text { Analysis of data and applications } & 18\end{array}$

$\begin{array}{lll}2.3 .1 & \text { The issue of unitarity } & 19\end{array}$

3 Leading twists and the $d=2$ limit 20

4 State decouplings at the Ising point $\quad 24$

4.1 Counting of quasi-primary states on the $d=2$ unitarity boundary and Virasoro null vectors 25

4.2 Numerical spectrum near the Ising point and decoupling of states for $2 \leq d \leq 329$

$\begin{array}{lll}5 & \text { Conclusions } & 31\end{array}$

$\begin{array}{ll}\text { A Numerical methods } & 31\end{array}$

\section{Introduction}

The study of conformal invariance in two dimensions, following the fundamental paper [1], has led to a deep understanding of non-perturbative phenomena in massless quantum field theories and the exact solution of many models with countless physical applications [2].

Conformal invariance above two dimensions has long been considered of limited help in solving non-perturbative physics due to the lack of an analog of the infinite-dimensional Virasoro algebra. This implies among other things the existence of infinite conserved local currents, and the integrability of the theory (in principle, at least). A new perspective has recently emerged due to the success of the conformal bootstrap approach above two dimensions [3], that provided many remarkable non-perturbative results, both numerical and analytic, on critical exponents and other conformal data, most notably for the Ising model in three-dimensions [4].

In view of these developments, the interplay between conformal invariance in two and higher dimensions requires some better understanding and a convenient approach is that of studying the dependence of theories on the continuous dimension $d \geq 2$. For example, the decomposition of correlators in 'conformal partial waves' [5, 6], a basic ingredient for setting up the bootstrap, depends smoothly on $d$. On the contrary, the algebraic structures 
related to representations of the Virasoro algebra and the existence of minimal models with reduced sets of states are very specific to $d=2$. Thus, a rather general question is whether any of these structures can be generalized to $d>2$ (and how). More specifically, we might ask:

- How the reduced sets of states of the $d=2$ Ising minimal model extends when the dimension is deformed to $d>2$ ?

- How the vast degeneracy of scaling dimensions of $d=2$ conformal fields breaks down for $d>2$ ?

- Are the $d>2$ conformal theories sitting on the boundary of the unitary region 'minimal' in some sense?

- In particular, do analogs of Virasoro null vectors exist for $d>2$ in the form of projection of states?

In this paper, we describe the numerical solution of the simplest one-correlator conformal bootstrap for thirteen values of $4>d>2$, directly improving the earlier works [7,8]. For each $d$ value, we use the SDPB program [9] for finding the boundary of the unitary region and then run the Extremal Functional Method [10, 11] to solve the truncated bootstrap equations along this boundary. We first reproduce the $d=3$ data and patterns of the 2014 paper by El-Showk et al. [12] and then extend to other dimensions with similar (high) precision. As in that paper, the Ising criticality is identified by the point with smallest value of central charge along the boundary, where also a kink is present. The use of the Extremal Functional Method greatly reduces the numerical work and allows for a better resolution of subleading fields, which are then compared with the advanced three-correlator bootstrap results of refs. $[4,13,14]$.

In section two, we present these checks at $d=3$ and then proceed to describe the conformal dimensions of six best identified low-lying fields $\mathcal{O}$, respectively $\sigma, \epsilon, \epsilon^{\prime}$ for spin $\ell=0, T^{\prime}$ for $\ell=2$ and $C, C^{\prime}$ for $\ell=4$, and the relative structure constants $f_{\sigma \sigma \mathcal{O}}$, for the values:

$$
d=3.75,3.5,3.25,2.75,2.5,2.25,2.2,2.15,2.1,2.05,2.01,2.00001 \text {. }
$$

These conformal data are then expressed as polynomials in $y=4-d$ obtained by least chisquare fits and then compared with earlier bootstrap results in non-integer dimension $[7,8]$, the epsilon expansion of $\lambda \phi^{4}$ theory [15-17], and Monte Carlo simulations [18]. We thus obtain a description of Ising criticality in continuous dimension $4>d \geq 2$ that can be useful for many applications, such as, for example, in developing some conjectures on universality classes $[19,20]$ and as a benchmark for testing resummations of the epsilon expansion [15-17, 21].

We remark that the six low-lying states depend smoothly on the dimension $d$ and their behavior does not allow to answer any of the previous questions on the interplay between $d=2$ and $d>2$. As is well known, degeneracies, null vectors and other features of Virasoro representations involve the higher part of the $d=2$ conformal spectrum: unfortunately, this is not described accurately enough in our numerical setting. 
Nonetheless, some hints of the $d$-dependent changes can be observed. In section three, we describe the spectrum of least-dimensional fields for each spin value $\ell$, the so called leadingtwists. Their dimension $\Delta_{\ell}=d-2+\ell+\gamma_{\ell}$ include a large classical part and a small anomalous dimension $\gamma_{\ell}$, whose asymptotic behavior in $\ell$ is given by:

$$
\lim _{\ell \rightarrow \infty} \gamma_{\ell}=2 \gamma_{\sigma}, \quad \Delta_{\ell}=d-2+\ell+\gamma_{\ell}, \quad \Delta_{\sigma}=\frac{d-2}{2}+\gamma_{\sigma},
$$

where $\Delta_{\sigma}$ is the dimension of the Ising spin. The numerical bootstrap gives values of $\Delta_{\ell}$ that are unstable, oscillating between $\gamma_{\ell}=0$ and $\gamma_{\ell} \neq 0$ within the range of $\Delta_{\sigma}$ values identifying the Ising point - a known feature of the Extremal Functional Method [13]. Yet, the non-vanishing $\gamma_{\ell}$ values match rather well the precise $d=3$ values found by the threecorrelator bootstrap [13], and give us confidence for analyzing $\gamma_{\ell}(d)$ for $3>d>2$ and $4 \leq \ell \leq 20$. For any dimension $d>2.2$, the $\gamma_{\ell}(d)$ obey the asymptotic limit (1.2) and also satisfy the Nachtmann theorem [22-24], i.e. they form a curve in $\ell$ that is monotonically increasing and convex.

However, at $d \leq 2.2$ such a behavior is lost and all anomalous dimensions converge to the values $\gamma_{\ell}=0$, that pertain to higher-spin conserved currents fitting the Virasoro tower of the identity field $I$ in the $d=2$ Ising model. The subleading-twist fields analogously converge to $\gamma_{\ell}=1$ for entering the tower of the energy field $\epsilon$. This result establishes that $d \sim 2.2$ is the dimension at which conformal theories actually acquire the $d>2$ structure, and that the transition between the $d>2$ and $d=2$ regimes takes place in the region $2.2>d>2$.

In section four, we analyze the fate of the Virasoro null vectors as the dimension is increased above two. We start in $d=2$ by recalling the Zamolodchikov counting of quasiprimary fields in the minimal models [25], and use it to formulate a necessary condition for the occurrence of null vectors. This indicates when a bootstrap channel decouples as the Ising point is approached from $\Delta_{\sigma} \geq 1 / 8$ along the unitarity boundary, corresponding to a proper null vector of the Ising model.

Among the low-lying states numerically accessible at $d=2$, we find that the simplest $\ell=2$ null vector of the Ising energy field $\epsilon=\phi_{1,3} \sim \phi_{2,1}$ (in the Kac table [1]) is clearly seen, while the higher ones are blurred. Following the evolution of this state in the bootstrap spectrum for $d>2$ and $\Delta_{\sigma} \geq \Delta_{\sigma}^{\text {Ising }}$, we find that it keeps decoupling at the Ising point till $d=3$ where it had been observed before [12]. This is a very interesting and encouraging result w.r.t. the questions raised at the beginning, suggesting that $d>2$ Ising conformal theory could be characterized by specific decouplings of states. However, some words of caution are necessary, because a single occurrence does not prove the existence of a pattern.

Finally, in section five we draw our conclusions and in appendix A we discuss the numerical procedures employed in this work.

\section{Precise critical exponents and structure constants as functions of di- mension}

\section{1 $\quad$ Method and $d=3$ checks}

The first step of our analysis is to reproduce the results of the one-correlator bootstrap at $d=3$ by El-Showk et al. [12, 26]. To this effect, we consider precisely the same setting, 
truncating the functional space of the bootstrap equation to 153 and 190 components. We use the SDPB algorithm [9] to find the unitarity boundary in the $\left(\Delta_{\sigma}, \Delta_{\epsilon}\right)$ plane and solve the bootstrap equations on this boundary by the Extremal Functional Method [10, 11]. We obtain the plots of conformal dimensions as a function of $\Delta_{\sigma}$ that indeed reproduce the patterns shown in the figures 7 to 16 of section 3 in ref. [12]. The numerical methods employed here are freely available and have been adapted to varying dimension; the details of their implementation are discussed in appendix A. A key feature of our approach is the gain of computational speed provided by the Extremal Functional Method that allowed us to study several values of $d$ with reasonable effort.

A crucial point is the determination of the value of $\Delta_{\sigma}$ on the unitarity boundary corresponding to the Ising model, which then fixes the other conformal data. In earlier works, this has been identified by the point where the boundary in the $\left(\Delta_{\sigma}, \Delta_{\epsilon}\right)$ plane has a kink and by the minimum of the central charge $c$. These two features are shown in figure 1(a) and 1(b), respectively. Let us discuss this issue in detail.

The analysis of ref. [12] identified the Ising point by the minimum of $c$ in figure 1(b): the authors noted that by improving the bootstrap precision from 153 (light blue points) to 190 (dark blue points) and 231 (not shown) components, the minimum moves to the left and eventually stays within the range $\Delta_{\sigma}=0.51814-0.51817$, from which they extracted the precise value $\Delta_{\sigma}=0.518154(15)$ and the estimated error.

In this work, we adopt a slightly different approach that is convenient for the other dimensions $d$ as well: we consider both the $c$ minimum and the kink position. In figure 1(b), the former is placed to the right of $\Delta_{\sigma}=0.518170$, as said; in figure 1(a), the latter is identified by the crossing of the left and right tangents to the boundary, around $\Delta_{\sigma}=$ 0.518150. As expected, the kink and the minimum are not exactly at the same point, thus we should choose a value in between, and use the mismatch as an estimate of the error. We thus take an error range of four data points as indicated by the gray area in figure 1(a). The values of $\Delta_{\epsilon}$ and $c$ and their errors are found by reading the corresponding ranges on the $y$ axes of figure $1(\mathrm{a})$ and $1(\mathrm{~b})$.

In summary, our identification of the Ising point gives the values $(d=3)$ :

$$
\begin{aligned}
\Delta_{\sigma} & =0.518155(15), \\
\Delta_{\epsilon} & =1.41270(15), \\
c & =0.946535(15) .
\end{aligned}
$$

These results are very similar to those of ref. [12], (see tables 1 and 2 for a comparison); our errors are slightly worse owing to the conventions just explained. The enhanced $c$ error in figure 1 (b) will be clarified later. It is very interesting and reassuring that we find consistent results by using different numerical routines.

Let us remark that the value of $c$ is determined by the structure constant $f_{\sigma \sigma T}$ of the stress tensor $T$ using the formula:

$$
f_{\sigma \sigma T}^{2}=\frac{d \Delta_{\sigma}^{2}}{4(d-1) c}
$$




\begin{tabular}{|c|c|l|l|l|}
\hline$\Delta(d=3)$ & $\ell$ & this work & 3 - correlator & 1 - correlator \\
\hline$\Delta_{\sigma}$ & 0 & $0.518155(15)$ & $0.5181489(10)$ & $0.518154(15)$ \\
$\Delta_{\epsilon}$ & 0 & $1.41270(15)$ & $1.412625(10)$ & $1.41267(13)$ \\
$\Delta_{\epsilon^{\prime}}$ & 0 & $3.8305(15)$ & $3.82968(23)$ & $3.8303(18)$ \\
$\Delta_{\epsilon^{\prime \prime}}$ & 0 & $7.01(5)$ & $6.8956(43)$ & \\
\hline$\Delta_{T^{\prime}}$ & 2 & $5.505(10)$ & $5.50915(44)$ & $5.500(15)$ \\
$\Delta_{T^{\prime \prime}}$ & 2 & $7.25(55)$ & $7.0758(58)$ & $\approx 7$ \\
\hline$\Delta_{C}$ & 4 & $5.026(4)$ & $5.022665(28)$ & \\
$\Delta_{C^{\prime}}$ & 4 & $6.67(23)$ & $6.42065(64)$ & \\
$\Delta_{C^{\prime \prime}}$ & 4 & $7.45(5)$ & $7.38568(28)$ & \\
\hline
\end{tabular}

Table 1. Comparison of $d=3$ conformal dimensions of low-lying fields with earlier results of one-correlator [12] and three-correlator [13] bootstrap.

The central charge is normalized to $c=1$ for the free bosonic theory and the structure constants are defined within the conventions of the 3-correlator study [13], differing from those of the 1-correlator work [12] by the factor:

$$
\left(f_{\sigma \sigma \mathcal{O}_{\ell}}\right)_{3-\text { corr }}^{2}=\left(f_{\sigma \sigma \mathcal{O}_{\ell}}\right)_{1-\text { corr }}^{2} \frac{\left(\frac{d-2}{2}\right)_{\ell}}{(d-2)_{\ell}}
$$

where $\ell$ is the spin of the field $\mathcal{O}_{\ell}$ and $(x)_{\ell}$ is the Pochhammer symbol.

The solution of the bootstrap equations on the unitarity boundary yields a spectrum of conformal dimensions $\Delta_{\ell, i}$ as functions of the varying parameter $\Delta_{\sigma}$, divided into sectors of spin $\ell=0,2,4, \ldots$ and numbered by increasing size, $i=1,2, \ldots$ As said, our results for these curves are almost identical to those of ref. [12] and are not redrawn here. The data for the Ising model are obtained by reading the values of the curves at the point $\Delta_{\sigma}$ given in (2.1); the corresponding errors are given by the maximal curve variation within the $\Delta_{\sigma}$ error range, as in the case of $\Delta_{\epsilon}$ in figure 1(a). The subleading fields, $\Delta_{i, \ell}, i=2, \ldots$, show larger fluctuations within this range, because the spectrum changes considerably around the Ising point and the errors become larger and larger. Of course, we report the best data obtained by the bootstrap truncated to 190 components; the comparison with the 153-components data is sometimes used as an estimate of systematic errors.

Our results for first few low-lying dimensions are shown in table 1: they are slightly better than those of ref. [12], owing to the improved numerical algorithms, and are then compared with the three-correlator bootstrap results [13], basically one order of magnitude more precise. The analogous comparison of structure constants is given in table 2 .

The analysis of the data in the two tables let us to conclude that there are six fields, $\mathcal{O}=\sigma, \epsilon, \epsilon^{\prime}, T^{\prime}, C, C^{\prime}$ of spin $\ell=0,2,4$, whose dimensions $\Delta_{\mathcal{O}}$ and structure constants $f_{\sigma \sigma \mathcal{O}}$ are sufficiently precise to be worth analyzing in other dimensions $4>d>2$. Note that the structure constants $f_{\sigma \sigma \mathcal{O}}$ are sometimes better determined than the corresponding dimensions $\Delta_{\mathcal{O}}$. 


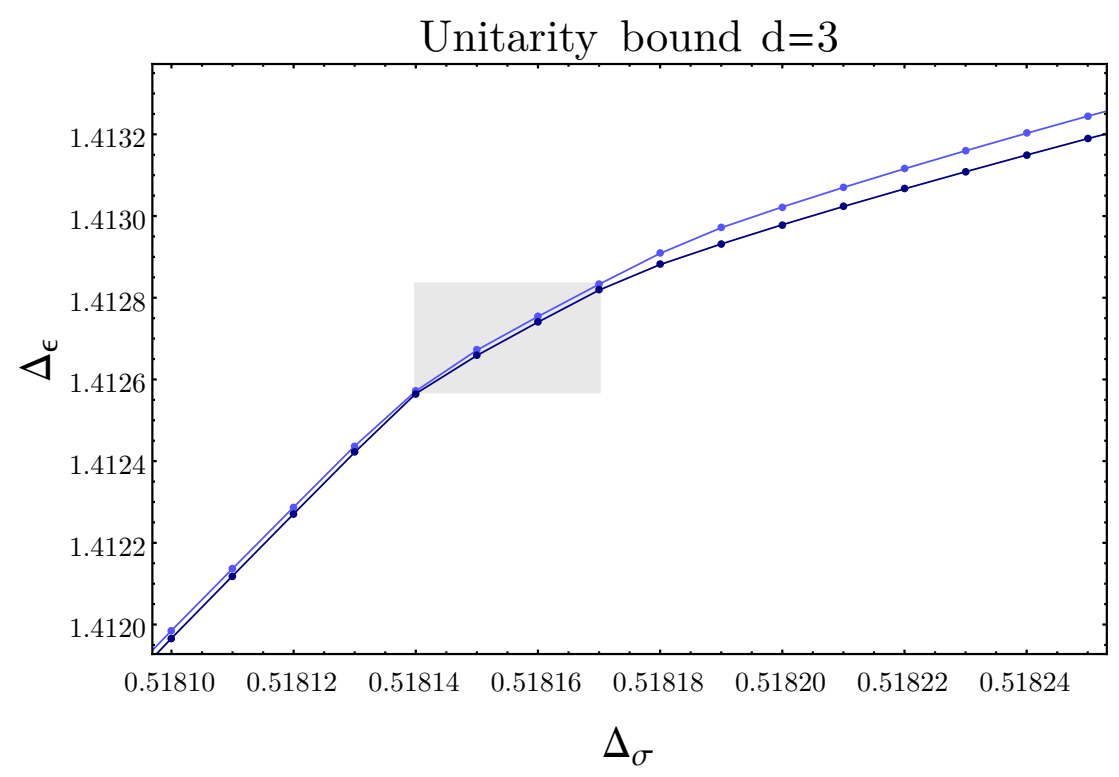

(a)

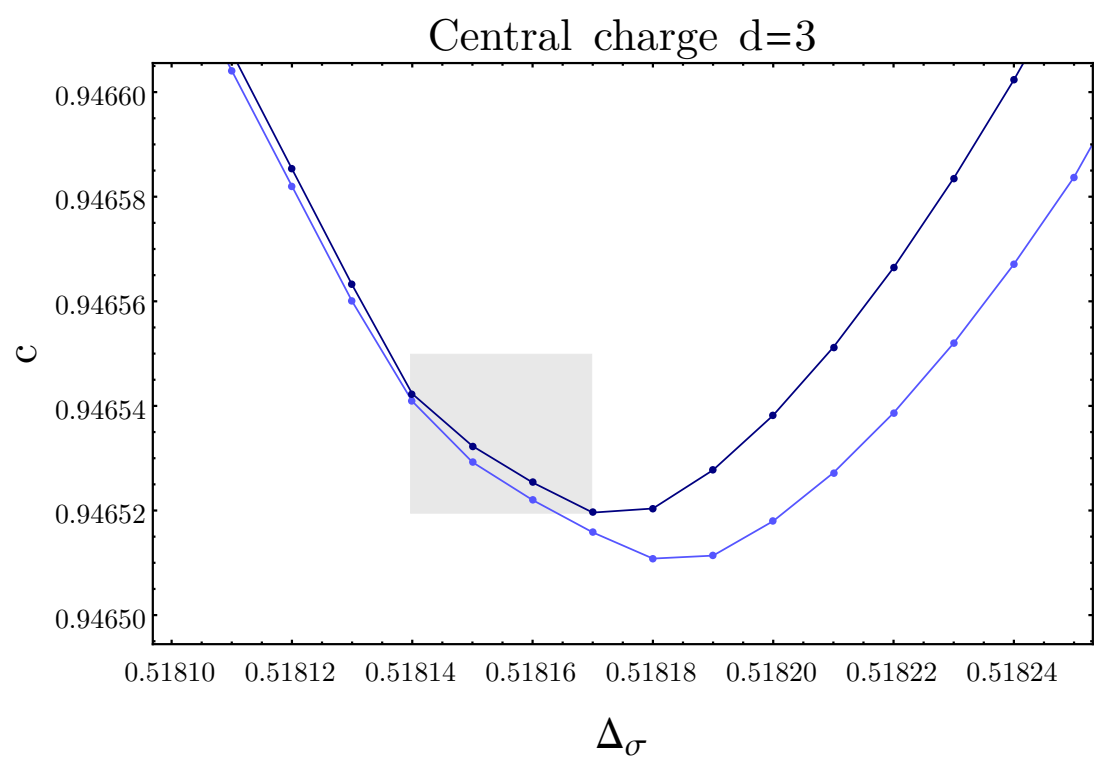

(b)

Figure 1. Determination of the Ising critical point for $d=3$ from: (a) kink on unitarity boundary; (b) minimum of central charge. The light (dark) blue lines correspond to bootstrap equations with 153 (190) components. The grey area indicates the estimated error.

\subsection{Polynomial fits of $4>d>2$ data and comparison with other approaches}

The determination of the Ising critical point on the unitarity boundary for dimensions $d=3,3.75,2.25,2.2,2.00001$ is shown in figure 2,3 : the plots are drawn on equal intervals $(0.0001,0.001,0.0001)$ of $\left(\Delta_{\sigma}, \Delta_{\epsilon}, c\right)$, respectively, for comparison, with the exception of the $d=2.2$ case, whose ranges are three times larger. We note that the minimum and kink are 


\begin{tabular}{|c|l|l|l|}
\hline$f_{\sigma \sigma \mathcal{O}}$ & this work & 3 - correlator & 1 - correlator \\
\hline$f_{\sigma \sigma \epsilon}$ & $1.051835(35)$ & $1.0518537(41)$ & $1.05184(4)$ \\
$f_{\sigma \sigma \epsilon^{\prime}}$ & $0.05300(5)$ & $0.053012(55)$ & $0.05301(5)$ \\
$f_{\sigma \sigma T}$ & $0.326142(7)$ & $0.32613776(45)$ & $0.326142(8)$ \\
$c$ & $0.946535(15)$ & $0.9463385(60)$ & $0.946534(11)$ \\
$f_{\sigma \sigma T^{\prime}}$ & $0.010575(15)$ & $0.0105745(42)$ & $0.01055(4)$ \\
$f_{\sigma \sigma C}$ & $0.065(5)$ & $0.069076(43)$ & \\
$f_{\sigma \sigma C^{\prime}}$ & $0.0020(5)$ & $0.0019552(12)$ & \\
\hline
\end{tabular}

Table 2. Comparison of $d=3$ structure constants of six low-lying fields with earlier results of one-correlator [12] and three-correlator [13] bootstrap (the values of $f_{\sigma \sigma T}$ and $c$ are related).

of comparable shape for $3 \geq d \geq 2.2$ and become sharper at 3.75 and at $d=2.00001$ again. The data shows that it is not always clear where the $c$ minimum moves as the precision of the bootstrap is improved (from 153 to 190 components) and that the kink position helps in the identification of the Ising point. The plots at $d=2.2$ with reduced scale is shown as an example of error determination in the region $2.2 \geq d \geq 2.01$, where our sampling of the unitary boundary is coarser. In all cases the error range is chosen to be no less than $2-3$ data points.

\subsubsection{Conformal dimensions}

Tables 3 summarize the determination of dimensions for the six low-lying fields at twelve values of dimension. One sees that the precision attained at $d=3$ is roughly maintained for $4>d \geq 3$, it decreases in going from $d=3$ to $d=2.01$ and then is good again at $d=2.00001$. The lower quality around $d=2.25$ is due to the reordering of the conformal towers of states for approaching the $d=2$ theory that will be discussed in section three. For $2.2 \geq d \geq 2.01$, the reduced data sampling also affects the results.

We now proceed to analyze the data in each of the columns of table 3 and study their dependence on spacetime dimension, starting with the case of $\Delta_{\sigma}$. We perform a least chisquare fit of the anomalous dimension $\gamma_{\sigma}=\Delta_{\sigma}-(d-2) / 2$ with a polynomial in $y=4-d$, and obtain the result:

$$
\begin{aligned}
& \gamma_{\sigma}(y)= 0.00955001 y^{2}+0.00764826 y^{3}+0.00091284 y^{4}-0.00024948 y^{5} \\
&+0.000296768 y^{6}, \\
& \operatorname{Err}\left(\gamma_{\sigma}\right)<0.0001 .
\end{aligned}
$$

The polynomial is of $(2-6)$-th order, i.e. it is supposed to start quadratically at $d=4$, as suggested by the epsilon expansion of $\lambda \phi^{4}$ theory (a linear term would not improve the fit). Moreover, it includes a sufficient number of terms for obtaining a reasonably small chi-square value. ${ }^{1}$ Since the fit is very precise, we do not plot the curve $\gamma_{\sigma}(y)$ but its difference with respect to the data points (see figure 4): one sees that $\gamma_{\sigma}(y)$ is determined with an overall error that is less than $10^{-4}$, although some individual points are far more

\footnotetext{
${ }^{1}$ Some digits in the coefficients of the polynomial (2.6) might be redundant for the achieved precision but are kept anyway.
} 


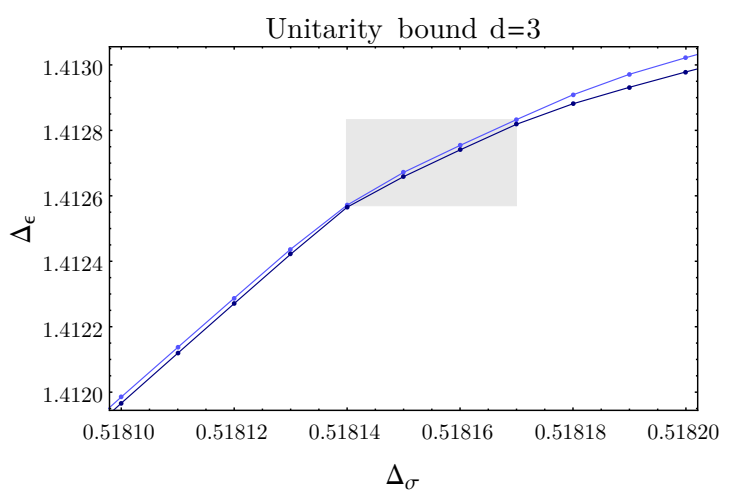

(a)

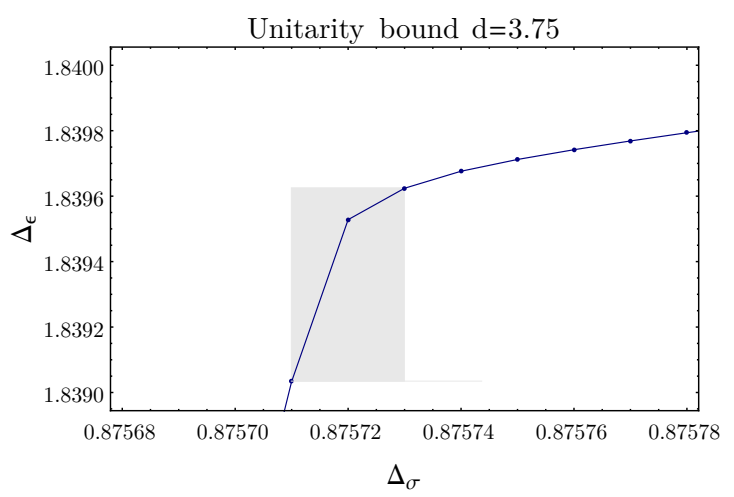

(c)

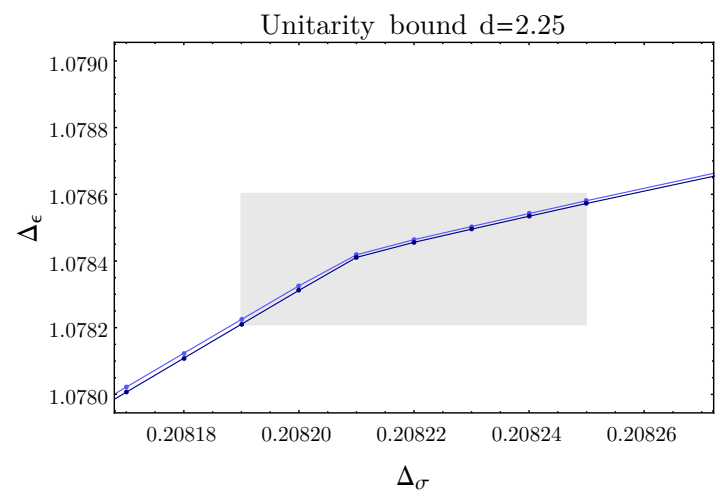

(e)

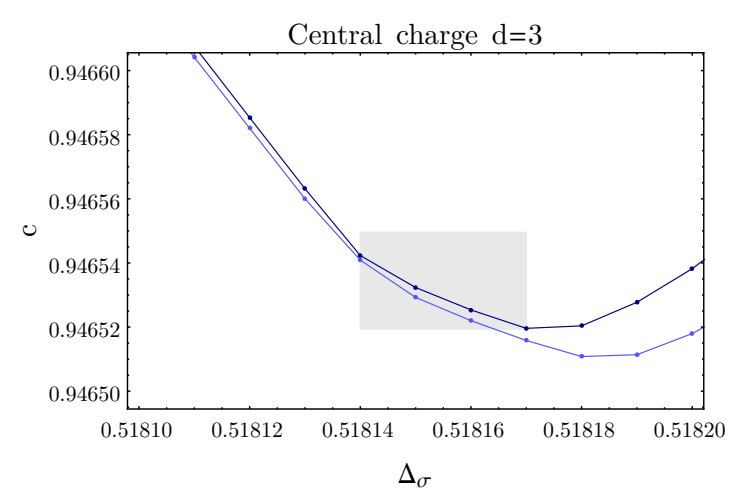

(b)

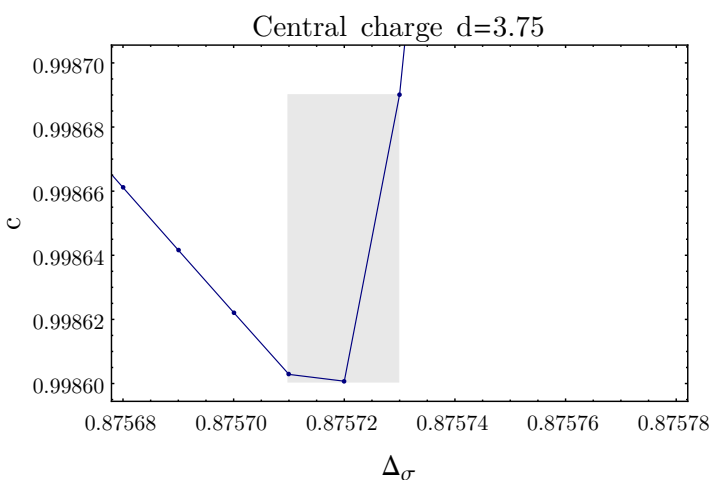

(d)

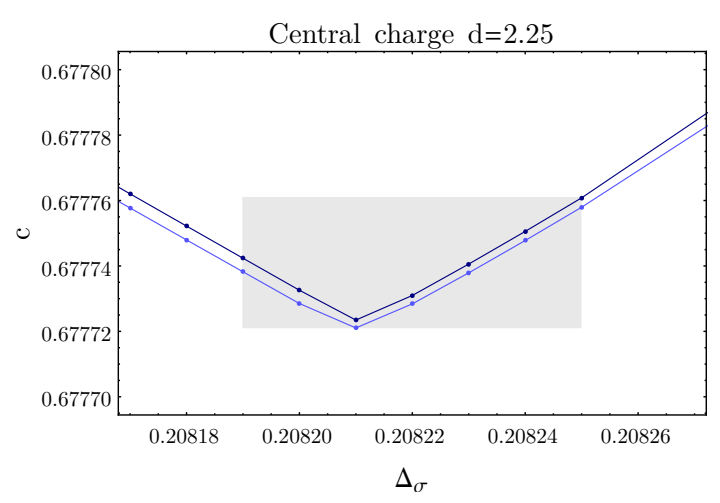

(f)

Figure 2. Determination of the Ising critical point for $d=3$ (rescaled view of figure 1 ), $d=3.75$ and $d=2.25$. As in figure 1, the light (dark) blue lines correspond to bootstrap equations with 153 (190) components and the grey area is the error.

precise. Since $\gamma_{\sigma}=O\left(10^{-1}\right)$, the relative error is less than $10^{-3}$. Note that the very good matching of the $d=2$ value is not imposed but obtained by the fit.

Equation (2.6) is one of the most interesting results of this work, expressing the conformal dimension $\Delta_{\sigma}$ as a function of $d$ with high precision, a long-sought goal of quantum field theory since the seventies [27]. Let us compare with the results of other methods. 


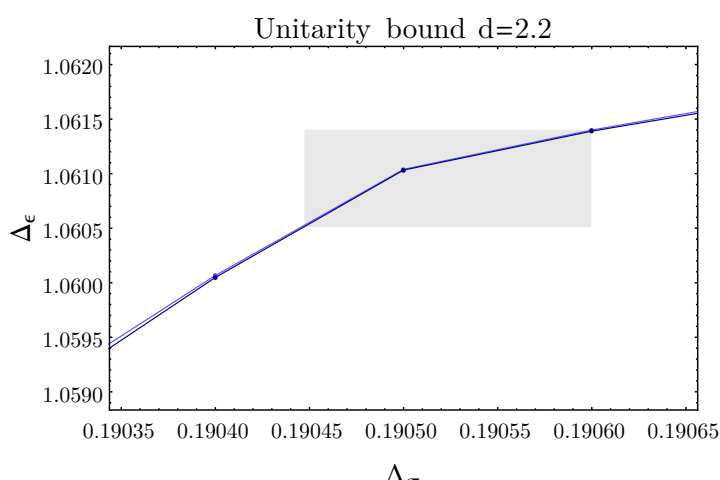

(a)

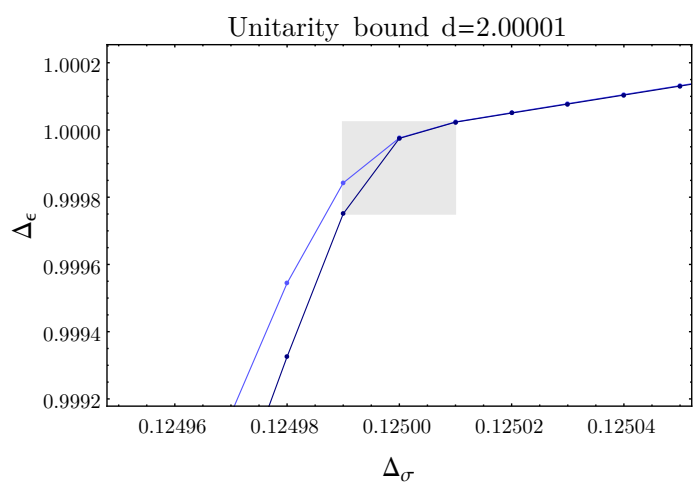

(c)

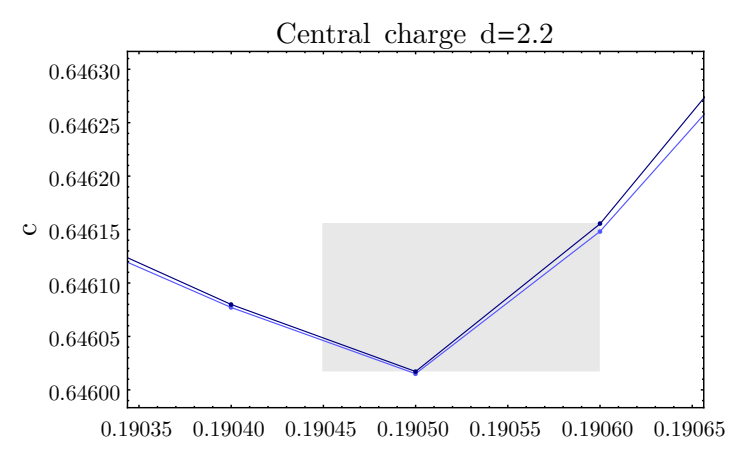

$\Delta_{\sigma}$

(b)

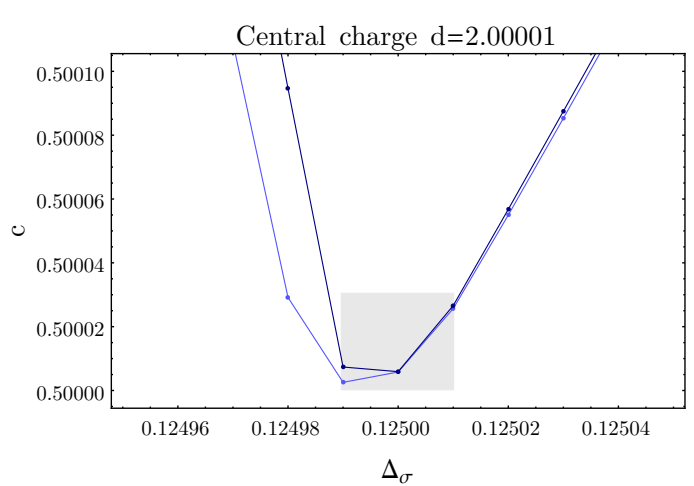

(d)

Figure 3. Determination of the Ising critical point for $d=2.2$ (smaller scale) and $d=2.00001$.

\begin{tabular}{|c|l|l|l|l|l|l|}
\hline$d$ & $\Delta_{\sigma}$ & $\Delta_{\epsilon}$ & $\Delta_{\epsilon^{\prime}}$ & $\Delta_{T^{\prime}}$ & $\Delta_{C}$ & $\Delta_{C^{\prime}}$ \\
\hline $\mathbf{4}$ & $\mathbf{1}$ & $\mathbf{2}$ & $\mathbf{4}$ & $\mathbf{6}$ & $\mathbf{6}$ & $\mathbf{8}$ \\
3.75 & $0.87572(1)$ & $1.83932(30)$ & $3.958(23)$ & $5.8622(14)$ & $5.750995(25)$ & $7.805(15)$ \\
3.5 & $0.753395(15)$ & $1.68851(31)$ & $3.921(11)$ & $5.734(6)$ & $5.50465(15)$ & $7.48(6)$ \\
3.25 & $0.633885(15)$ & $1.54638(18)$ & $3.8770(25)$ & $5.613(8)$ & $5.2625(15)$ & $7.145(35)$ \\
3 & $0.518155(15)$ & $1.41270(15)$ & $3.8305(15)$ & $5.505(10)$ & $5.026(4)$ & $6.67(23)$ \\
2.75 & $0.407465(35)$ & $1.2887(2)$ & $3.800(2)$ & $5.445(15)$ & $4.790(5)$ & $6.3(2)$ \\
2.5 & $0.30341(1)$ & $1.17625(15)$ & $3.797(1)$ & $5.455(25)$ & $4.574(9)$ & $5.78(13)$ \\
2.25 & $0.20822(3)$ & $1.0784(2)$ & $3.847(1)$ & $5.575(45)$ & $4.344(14)$ & $5.36(6)$ \\
2.2 & $0.19053(8)$ & $1.06095(45)$ & $3.864(4)$ & $5.685(35)$ & $4.325(15)$ & $5.29(4)$ \\
2.15 & $0.17333(8)$ & $1.04435(35)$ & $3.891(6)$ & $5.64(13)$ & $4.275(25)$ & $5.19(1)$ \\
2.1 & $0.14663(8)$ & $1.02855(45)$ & $3.9215(5)$ & $5.82(1)$ & $4.165(35)$ & $5.115(35)$ \\
2.05 & $0.14048(8)$ & $1.0134(7)$ & $3.9565(5)$ & $5.905(1)$ & $4.13(6)$ & $5.065(15)$ \\
2.01 & $0.12803(8)$ & $1.0011(17)$ & $3.990(1)$ & $5.9815(5)$ & $4.0144(1)$ & $5.0115(15)$ \\
2.00001 & $0.12500(1)$ & $0.99989(14)$ & $4.00015(20)$ & $6.0006(2)$ & $4.000055(10)$ & $5.00048(8)$ \\
$\mathbf{2}$ & $\mathbf{0 . 1 2 5}$ & $\mathbf{1}$ & $\mathbf{4}$ & $\mathbf{6}$ & $\mathbf{4}$ & $\mathbf{5}$ \\
\hline
\end{tabular}

Table 3. Dimensions of six low-lying states for $4>d>2$. The exact values for $d=2,4$ are given in bold. 


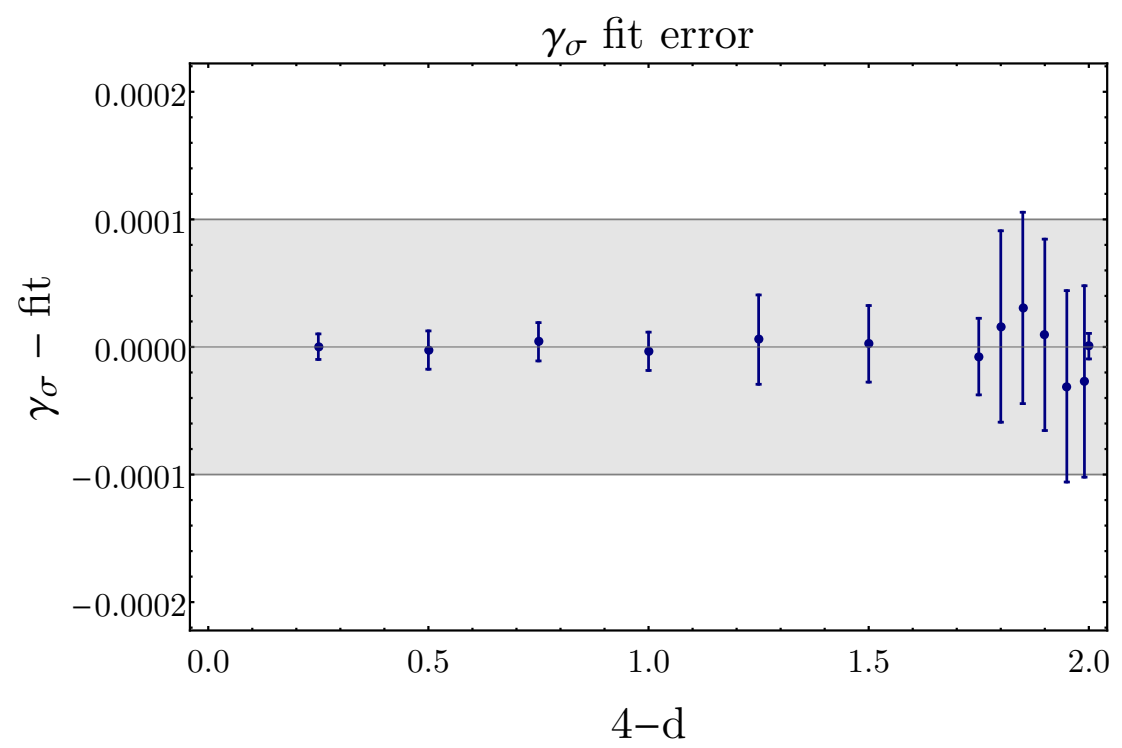

Figure 4. Quality of the polynomial fit for $\gamma_{\sigma}(4-d)$ bootstrap data in the range of dimensions $4>d \geq 2$. The maximal overall error of the fit is indicated by a grey band.

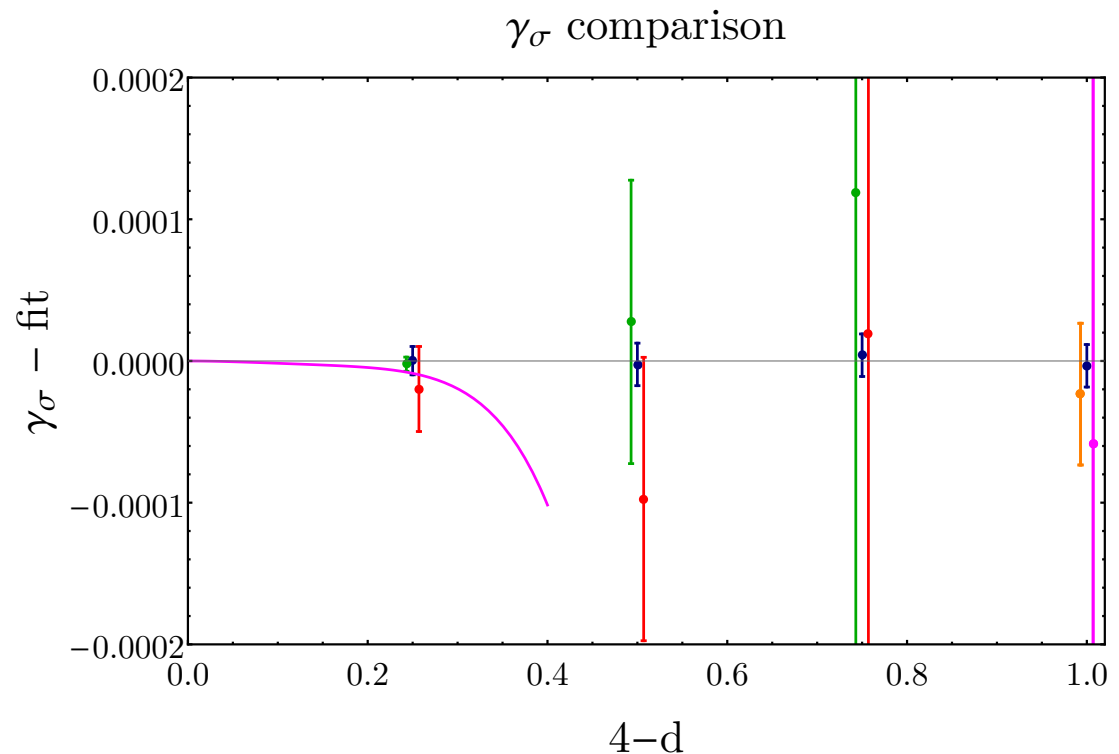

Figure 5. Comparison of $\gamma_{\sigma}$ bootstrap data (blue) with: i) Borel-resummed epsilon expansion (green) $[15,16]$ and ii) 3 -correlator bootstrap data (red) [8] for $d=3.75,3,5,3.25$ (the $d=3$ coarse data are omitted); iii) unresummed high-order epsilon expansion and resummed estimate at $d=3$ (magenta) [17] iv) Monte Carlo data at $d=3$ (orange) [18] (Note that data points are slightly displaced around the same $d$ value for improving readability). 
Figure 5 shows again the difference between data and fit (limited to the range $4>d \geq 3$ ), together with the following inputs:

- Green data points - Borel-resummed epsilon expansion of $\lambda \phi^{4}$ theory obtained in the nineties $[15,16]$ at $d=3.75,3.5,3.25$ (the $d=3$ value is less precise and is omitted); note the very good match at $d=3.75$.

- Magenta curve and data point — unresummed high-order epsilon expansion series [17]:

$$
\begin{aligned}
\gamma_{\sigma}(y)= & 0.00925925 y^{2}+0.009345 y^{3}-0.00416438 y^{4}+0.0128283 y^{5} \\
& -0.0406359 y^{6},
\end{aligned}
$$

and $d=3$ value obtained by Borel resummation.

- Orange data point - Monte Carlo result at $d=3$ [18].

- Red data points - three-correlator bootstrap at $d=3.75,3.5,3.25$ obtained in ref. [8]. ${ }^{2}$ In this approach, the Ising point is uniquely identified by a small unitarity island in the $\left(\Delta_{\sigma}, \Delta_{\epsilon}\right)$ plane; thus, these data provide a check for our kink-minimum criteria discussed before.

Finally, our results have been checked against the early bootstrap data ${ }^{3}$ of ref. [7], finding agreement within their uncertainties. Let us also quote the works [28-30] where other numerical and perturbative results for the Ising critical exponents have been discussed.

The comparison in figure 5 shows very good consistency of our $\gamma_{\sigma}(y)$ formula (2.6) with all other methods. Note incidentally that the unresummed epsilon expansion series (2.7) is extremely good up to $y=0.2$. Let us consider the following naive argument: if this series were asymptotic as the $\lambda \phi^{4}$ perturbative expansion at $d=4$ (no proof of this), the $n$-th term would grow as $O(n !)$ and the sixth-order unresummed series (2.7) would start diverging at about $y \sim 1 / 6$. The data in figure 5 are consistent with this estimate but actually show a milder behavior.

The best $d=3$ results from bootstrap, epsilon expansion and Monte Carlo are summarized in table 4 . In this table, some data are obtained from the critical exponents $\gamma, \nu, \omega$, by assuming the scaling relations $\gamma_{\epsilon}=2-1 / \nu, \gamma_{\sigma}=1-\gamma /(2 \nu)$ and $\Delta_{\epsilon^{\prime}}=d+\omega$, and simple independent error propagation.

The previous analysis of the conformal dimension as a function of $d$ is now repeated for the $\epsilon$ field, leading to the results in figure 6 . The part $(a)$ shows the difference between the data for the anomalous dimension $\gamma_{\epsilon}=\Delta_{\epsilon}-d+2$ and the fitting polynomial:

$$
\begin{array}{rlrl}
\gamma_{\epsilon}(y)= & 0.336000 y+0.0914812 y^{2}-0.0229152 y^{3}+0.00729869 y^{4} \\
& +0.000890045 y^{5}, & \operatorname{Err}\left(\gamma_{\epsilon}\right)<0.001
\end{array}
$$

\footnotetext{
${ }^{2}$ We thank C. Behan for providing his data to us.

${ }^{3}$ Nicely sent us by S. Rychkov.
} 


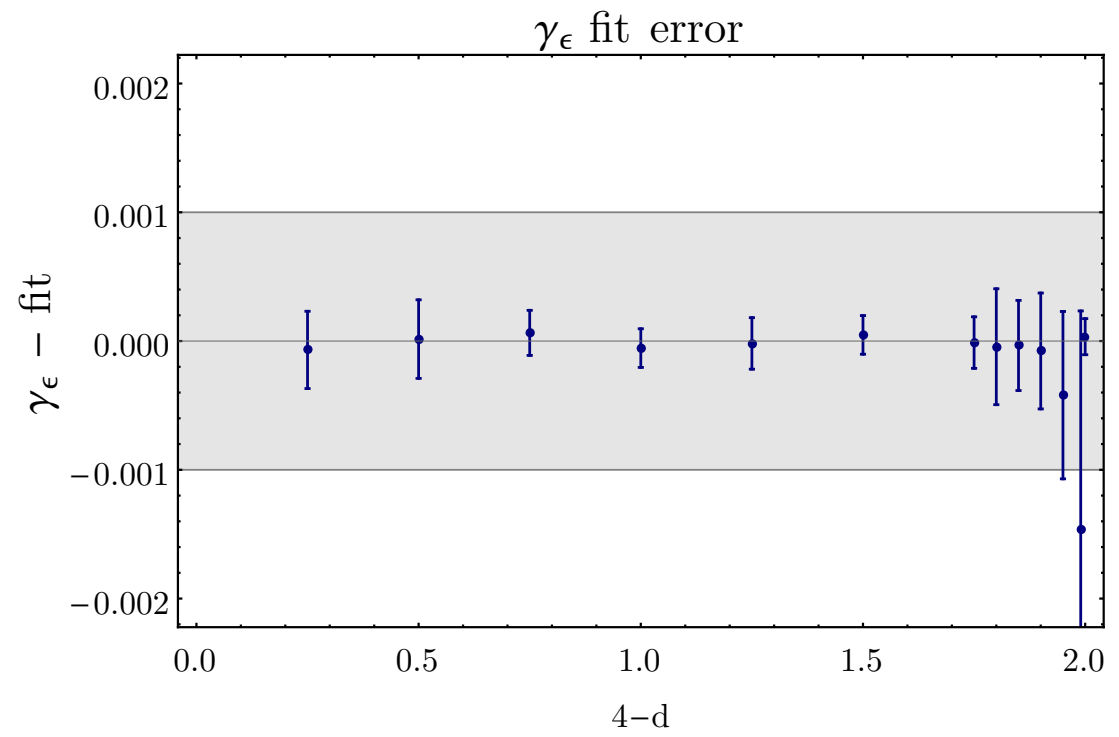

(a)

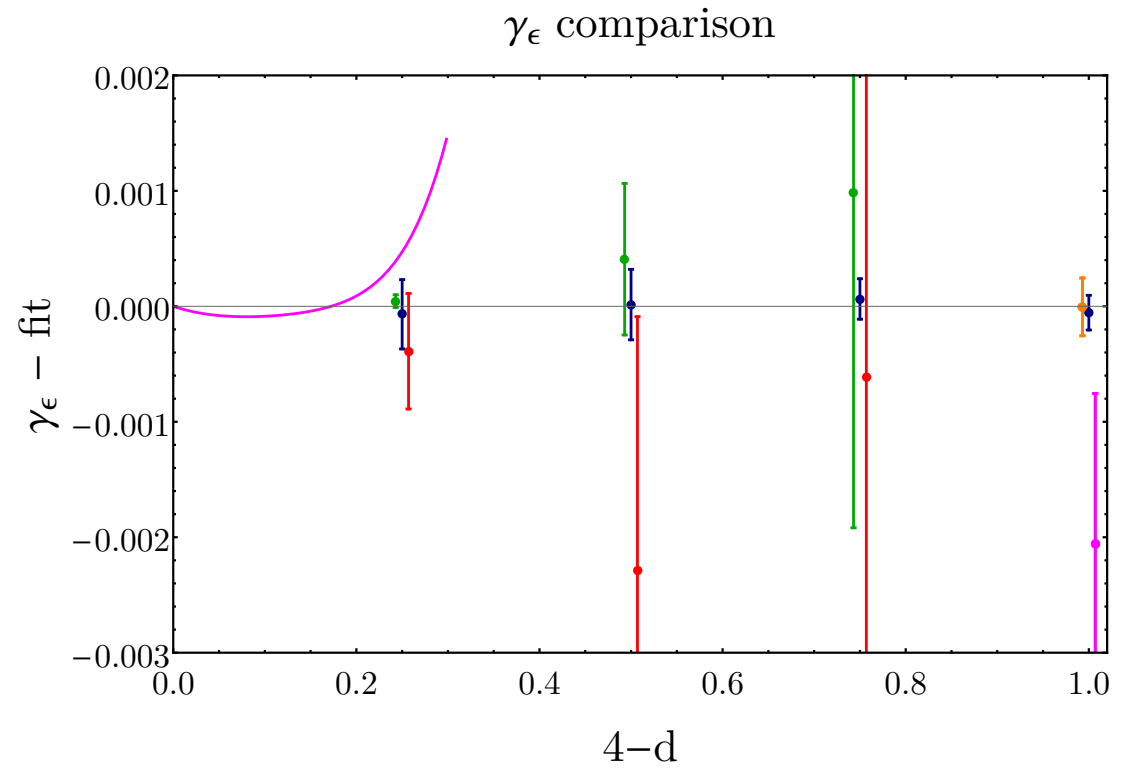

(b)

Figure 6. (a) Polynomial fit of $\gamma_{\epsilon}$ bootstrap data with overall error. (b) Comparison of bootstrap data (blue) with: resummed epsilon expansion (green); 3-correlator bootstrap (red); high-order epsilon expansion and resummed estimate at $d=3$ (magenta); Monte Carlo (orange) (references as in figure 5). 


\begin{tabular}{|c|l|l|l|l|}
\hline$d=3$ & this work & 3 - correlator & eps - expansion & Monte Carlo \\
\hline$\Delta_{\sigma}$ & $0.518155(15)$ & $0.5181489(10)$ & $0.5181(3)$ & $0.518135(50)$ \\
$\Delta_{\epsilon}$ & $1.41270(15)$ & $1.412625(10)$ & $1.4107(13)$ & $1.41275(25)$ \\
$\Delta_{\epsilon^{\prime}}$ & $3.8305(15)$ & $3.82968(23)$ & $3.820(7)$ & $3.832(6)$ \\
\hline
\end{tabular}

Table 4. Comparison of $d=3$ results for low-lying fields from bootstrap data of this work and the three-correlator analysis [13], resummed epsilon expansion [17] and Monte Carlo [18].

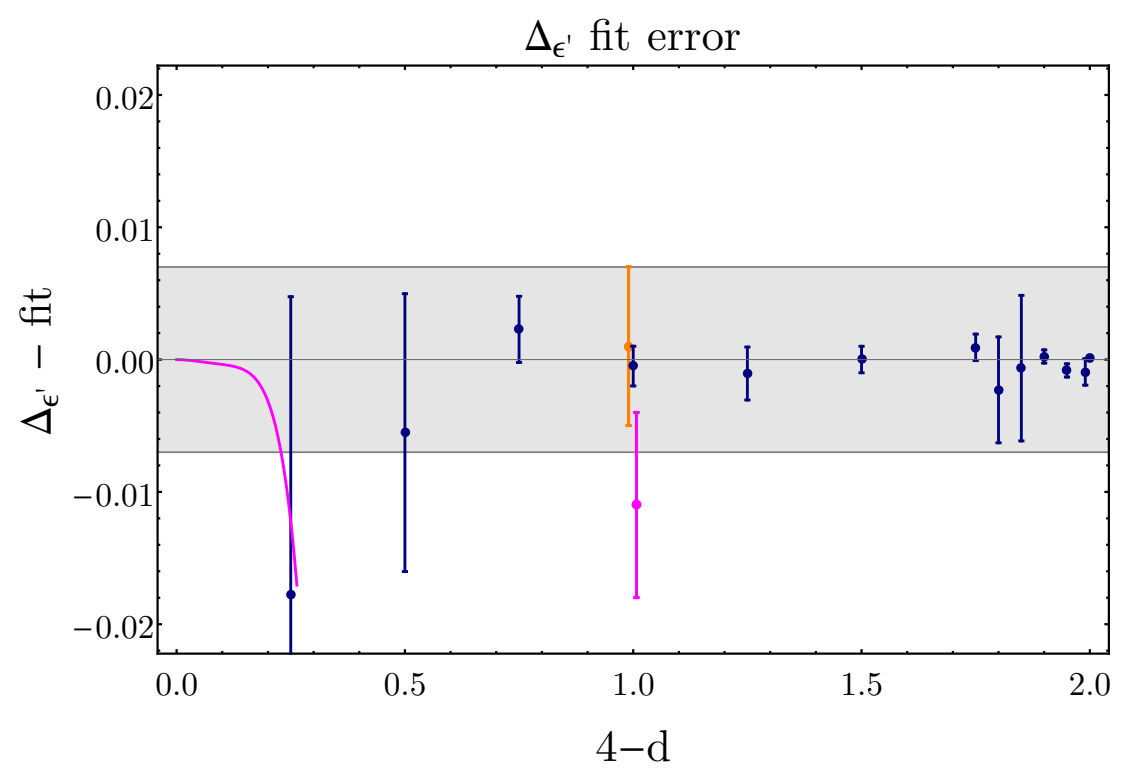

Figure 7. Fit of $\Delta_{\epsilon^{\prime}}$ bootstrap data (blue) with estimated error, high-order epsilon expansion with resummed estimate at $d=3$ (magenta) and Monte Carlo (orange) (references as in figure 5).

The fit assumes vanishing $\gamma_{\epsilon}$ at $d=4$ and involves five terms (the sixth one would have negligible coefficient). Note again the good match of the exact $d=2$ value $\gamma_{\epsilon}=1$. The overall error is less than $10^{-3}$ and the relative error is also less than $10^{-3}$ as in the case of $\gamma_{\sigma}$.

Figure 6(b) shows the comparison with other results, that are plotted using the same color code of figure 5. The unresummed epsilon expansion is [17]:

$$
\begin{aligned}
\gamma_{\epsilon}(y)= & 0.333335 y+0.117285 y^{2}-0.124528 y^{3}+0.30685 y^{4}-0.95125 y^{5} \\
& +3.57266 y^{6}, \quad \text { (Epsilon expansion) } ;
\end{aligned}
$$

this is also accurate for $y<0.2$. The consistency of all methods is again rather surprising.

The next quantity to analyze is the subleading scalar field $\epsilon^{\prime}$, that is related to the scaling exponent $\omega=\Delta_{\epsilon^{\prime}}-d$, widely analyzed in the literature. The least chi-square fit of our data gives:

$$
\begin{gathered}
\Delta_{\epsilon^{\prime}}(y)=4-0.530509 y^{2}+0.616593 y^{3}-0.334523 y^{4}+0.0794284 y^{5} \\
\operatorname{Err}\left(\Delta_{\epsilon^{\prime}}\right)<0.007 .
\end{gathered}
$$

It starts quadratically as the epsilon expansion (a linear term would worsen the fit). Figure 7 shows the difference between the data and the fit, together with the Monte Carlo 
results [18] and the epsilon expansion series [17]:

$$
\begin{gathered}
\Delta_{\epsilon^{\prime}}(y)=4-0.629625 y^{2}+1.61825 y^{3}-5.23513 y^{4}+20.7497 y^{5}-93.1109 y^{6}, \\
\text { (Epsilon expansion). }
\end{gathered}
$$

The two large errors in our $d=3.75,3.5$ data are probably due to the coarse sampling of the unitarity boundary, since the spectrum changes rapidly at these dimensions, as shown in figures 2(c), 2(d). Apart from this, the bootstrap results are rather good and consistent with those of the other methods. The overall error of the fit for $\Delta_{\epsilon^{\prime}}$ is estimated to be less than 0.007 (grey band). The $d=3$ results of different methods are again summarized in table 4 .

The analysis of the other fields $T^{\prime}, C, C^{\prime}$ can be done along the same lines. The resulting polynomials are as follows:

$$
\begin{aligned}
\Delta_{T^{\prime}}(y)= & 6-0.589135 y+0.210294 y^{2}-0.273167 y^{3} \\
& +0.157697 y^{4}, \\
\Delta_{C}(y)= & 6-y+0.0148373 y^{2}+0.233465 y^{3}-0.584595 y^{4}+0.642985 y^{5} \\
& -0.302706 y^{6}+0.0495537 y^{7} \\
\Delta_{C^{\prime}}(y)= & 8-0.518833 y-1.22242 y^{2}+0.729856 y^{3}-0.356502 y^{4} \\
& +0.0872808 y^{5}
\end{aligned}
$$

and the fits are shown in figure 8 . The errors are now bigger and vary considerably with $d$, thus it is not possible to give a $d$-independent bound for the error of the fit. We find:

\begin{tabular}{|c|c|c|c|}
\hline & $4>d>2.3$ & $2.3 \geq d \geq 2$ \\
\hline $\operatorname{Err}\left(\Delta_{T^{\prime}}\right)$ & $<0.03$ & $\sim 0.15$ \\
\hline $\operatorname{Err}\left(\Delta_{C}\right)$ & $<0.01$ & $\sim 0.10$ \\
\hline & $4>d>3$ & $3 \geq d \geq 2.5$ & $2.5>d \geq 2$ \\
\hline & \multicolumn{2}{|c|}{$\quad \sim 0.2$} & $<0.1$ \\
\hline
\end{tabular}

Comparison with the epsilon expansion series is possible in the case of the $\ell=4$ leading-twist $C[31-34]:^{4}$

$\Delta_{C}(y)=6-y+0.01296296 y^{2}+0.01198731 y^{3}-0.006591585 y^{4}, \quad$ (Epsilon expansion).

This field corresponds to a conserved current for both $d=4$ and $d=2$, thus it is convenient to plot its anomalous dimension $\gamma_{C}=\Delta_{C}-d+2$, that vanishes at both ends. In Figure 8(c), one sees that it grows slowly as $4>d>3$ and changes behavior at $d \sim 2.2$, where data fluctuate strongly. The behavior in this region will be further discussed in section three.

\footnotetext{
${ }^{4}$ The results of refs. [31-34] are obtained by a combination of epsilon expansion and analytic bootstrap approaches and might differ from the fully perturbative calculations of ref. [17], beyond the leading term. As such they might be closer to the numerical data for $y=O(1)$.
} 


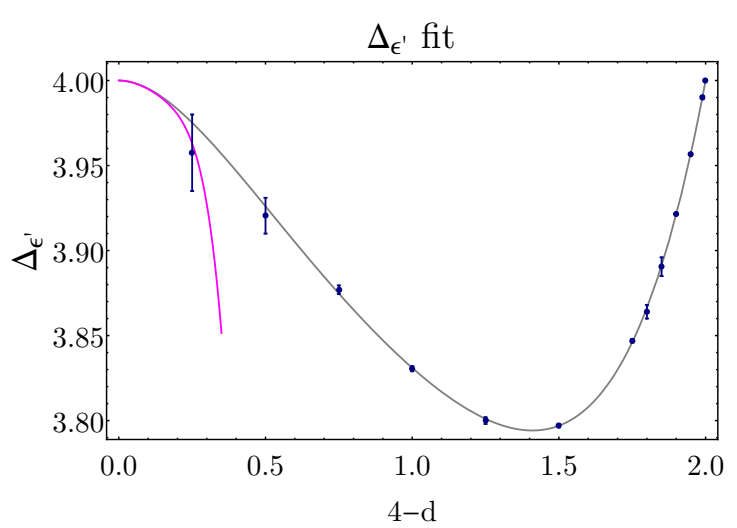

(a)

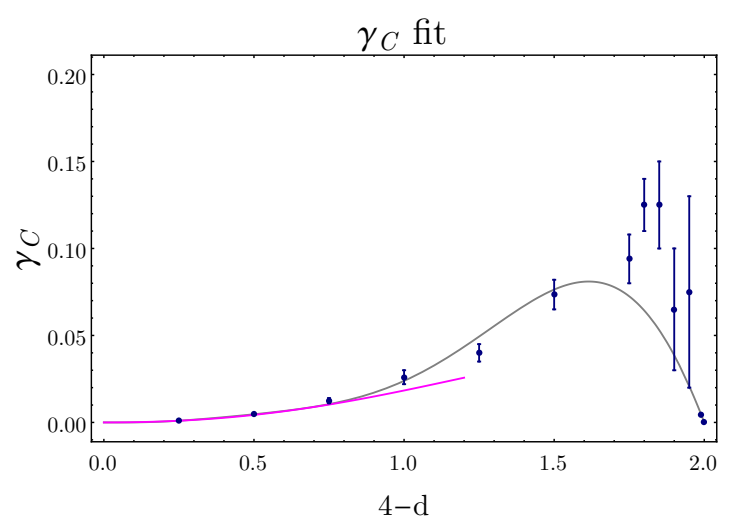

(c)

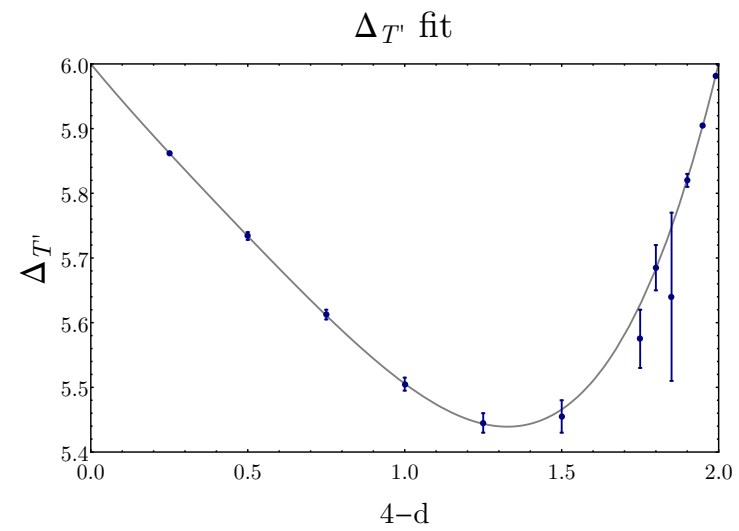

(b)

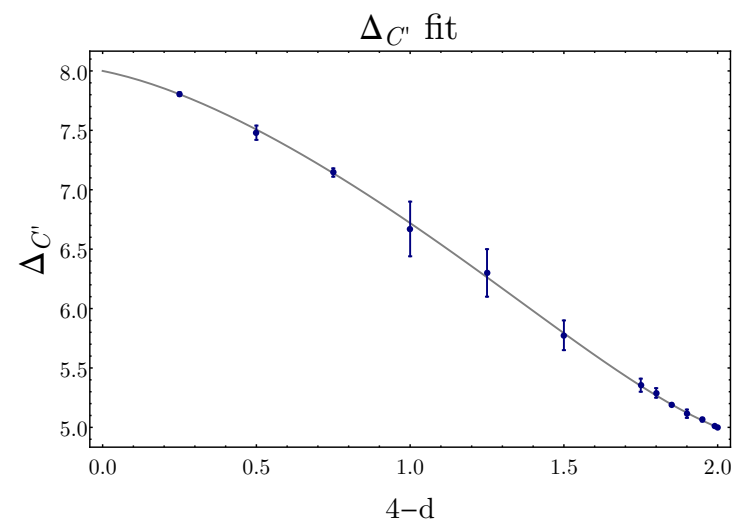

(d)

Figure 8. Polynomial fit of $\Delta_{\epsilon^{\prime}}, \Delta_{T^{\prime}}, \gamma_{C}, \Delta_{C^{\prime}}$, as functions of $y=4-d$. Figures (a) and (c) include the known epsilon expansion results.

\subsubsection{Structure constants}

The bootstrap data for the structure constants corresponding to the six fields discussed in the previous section are reported in table 5 . The exact values at $d=2,4$ are also included for comparison; for $d=4$ they are obtained from ref. [35],

$$
f_{\sigma \sigma \epsilon}=\sqrt{2}, \quad f_{\sigma \sigma C}=\frac{1}{\sqrt{35}}, \quad f_{\sigma \sigma \epsilon^{\prime}}=f_{\sigma \sigma T^{\prime}}=f_{\sigma \sigma C^{\prime}}=0,
$$

while for $d=2$ they are given in appendix B of ref. [36],

$$
f_{\sigma \sigma \epsilon}=\frac{1}{2}, \quad f_{\sigma \sigma \epsilon^{\prime}}=\frac{1}{64}, \quad f_{\sigma \sigma T^{\prime}}=\frac{3}{8^{3} \sqrt{10}}, \quad f_{\sigma \sigma C}=8 f_{\sigma \sigma T^{\prime}}, \quad f_{\sigma \sigma C^{\prime}}=\frac{1}{2^{8}} .
$$

The $d$-dependent polynomial fits for the structure constants are obtained as follows. The central charge is described by the polynomial:

$$
\begin{array}{rr}
c(y)= & 1-0.0173616 y^{2}-0.0133068 y^{3}-0.0385653 y^{4}+0.0310843 y^{5} \\
& -0.0196858 y^{6}+0.00436051 y^{7}, \quad \operatorname{Err}(c)<0.0007,
\end{array}
$$




\begin{tabular}{|c|l|l|l|l|l|l|}
\hline$d$ & $c$ & $f_{\sigma \sigma \epsilon}$ & $f_{\sigma \sigma \epsilon^{\prime}}$ & $f_{\sigma \sigma T^{\prime}}$ & $f_{\sigma \sigma C}$ & $f_{\sigma \sigma C^{\prime}}$ \\
\hline $\mathbf{4}$ & $\mathbf{1}$ & $\mathbf{1 . 4 1 4 2 1 3 6}$ & $\mathbf{0}$ & $\mathbf{0}$ & $\mathbf{0 . 1 6 9 0 3 1}$ & $\mathbf{0}$ \\
3.75 & $0.998645(45)$ & $1.34595(20)$ & $0.02725(25)$ & $0.00565(5)$ & $0.139775(25)$ & $0.00140(2)$ \\
3.5 & $0.992277(17)$ & $1.26142(18)$ & $0.04432(10)$ & $0.0091(1)$ & $0.112(2)$ & $0.0018(3)$ \\
3.25 & $0.976872(16)$ & $1.16283(7)$ & $0.05226(7)$ & $0.01061(17)$ & $0.086(4)$ & $0.0019(2)$ \\
3 & $0.946535(15)$ & $1.051835(35)$ & $0.05300(5)$ & $0.010575(15)$ & $0.065(5)$ & $0.0020(5)$ \\
2.75 & $0.893275(15)$ & $0.929385(35)$ & $0.04794(8)$ & $0.00901(6)$ & $0.048(4)$ & $0.00235(15)$ \\
2.5 & $0.80711(1)$ & $0.7963025(45)$ & $0.03885(2)$ & $0.006675(25)$ & $0.0325(25)$ & $0.00285(25)$ \\
2.25 & $0.677724(2)$ & $0.653111(16)$ & $0.027375(35)$ & $0.00394(14)$ & $0.0195(15)$ & $0.0035(2)$ \\
2.2 & $0.64609(7)$ & $0.62333(6)$ & $0.0245(5)$ & $0.00352(7)$ & $0.0185(35)$ & $0.00375(25)$ \\
2.15 & $0.61243(8)$ & $0.59313(8)$ & $0.0225(5)$ & $0.0025(5)$ & $0.017(3)$ & $0.00385(15)$ \\
2.1 & $0.5768(1)$ & $0.56249(7)$ & $0.02018(8)$ & $0.00265(5)$ & $0.0155(25)$ & $0.00395(15)$ \\
2.05 & $0.53935(15)$ & $0.53143(8)$ & $0.01785(5)$ & $0.0023(1)$ & $0.0135(25)$ & $0.0039(1)$ \\
2.01 & $0.5082(3)$ & $0.5058(6)$ & $0.01605(5)$ & $0.001925(25)$ & $0.0155(1)$ & $0.00392(1)$ \\
2.00001 & $0.500015(15)$ & $0.4999975(45)$ & $0.0156225(35)$ & $0.0018520(5)$ & $0.0148235(15)$ & $0.003904(1)$ \\
$\mathbf{2}$ & $\mathbf{0 . 5}$ & $\mathbf{0 . 5}$ & $\mathbf{0 . 0 1 5 6 2 5 0}$ & $\mathbf{0 . 0 0 1 8 5 2 9 0}$ & $\mathbf{0 . 0 1 4 8 2 3 2}$ & $\mathbf{0 . 0 0 3 9 0 6}$ \\
\hline
\end{tabular}

Table 5. Structure constants of six low-lying states for $4>d>2$. The exact values for $d=2,4$ are given in bold. The normalization conventions are as in ref. [13] (cf. eq. (2.5).

and the fit error is shown in figure 9. This quantity is very precise with an error less than $10^{-3}$. The high-order polynomial is necessary to obtain a good chi-square value: lowerorder polynomials would let the data oscillate out of the fit as $d$ varies. A remnant of this behavior is still present in figure 9 , and was also observed in early bootstrap data [7]. In order to comply with the fit, we slightly enlarged the error estimate of $c$ for some $d$ values, as shown in figure 1(b), for example.

The epsilon expansion of this quantity is known to fourth order [31, 32],

$$
c(y)=1-0.0154321 y^{2}-0.0266347 y^{3}-0.0039608 y^{4}, \quad \text { (Epsilon expansion) }
$$

and its relation with the data is also shown in figure 9 .

The structure constant $f_{\sigma \sigma \epsilon}$ is another precise result of the bootstrap. Its polynomial fit is:

$$
\begin{aligned}
f_{\sigma \sigma \epsilon}(y)= & 1.41421-0.235735 y-0.164305 y^{2}+0.0631842 y^{3}-0.0371191 y^{4} \\
& +0.0137454 y^{5}-0.00214024 y^{6}, \quad \operatorname{Err}\left(f_{\sigma \sigma \epsilon}\right)<0.0002
\end{aligned}
$$

while the known epsilon expansion is [31, 32],

$$
f_{\sigma \sigma \epsilon}(y)=1.41421-0.235702 y-0.168047 y^{2}+0.100996 y^{3}
$$

(Epsilon expansion).

Note again the close values of the first few coefficients of the two series. 


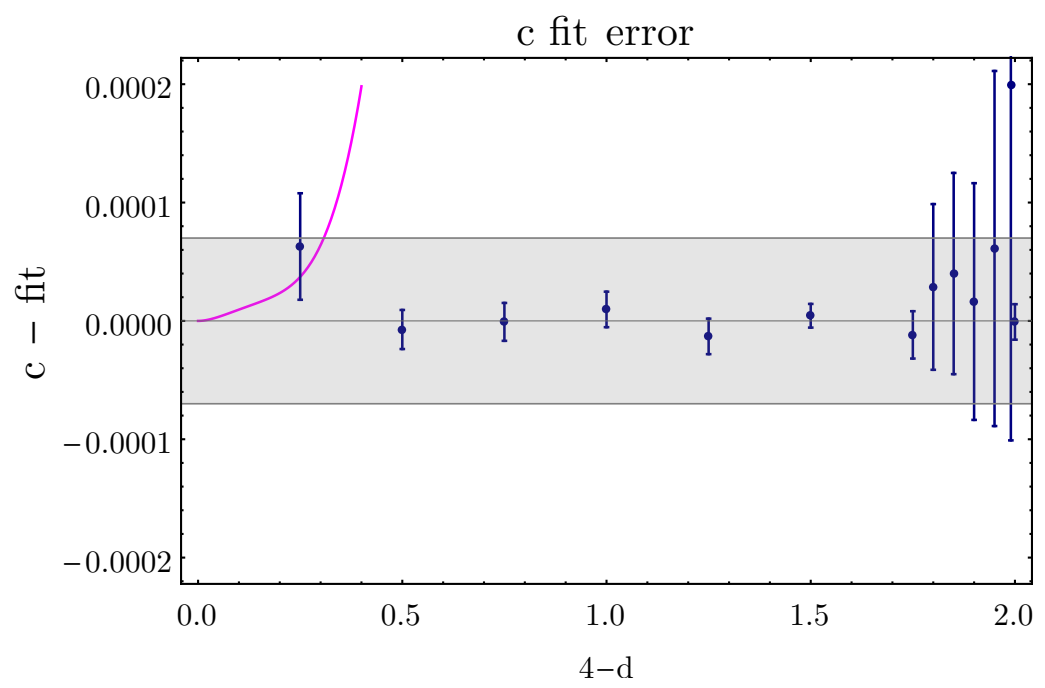

Figure 9. Fit of central charge $c$ bootstrap data (blue) with overall error (gray) and epsilon expansion (magenta) [31, 32].

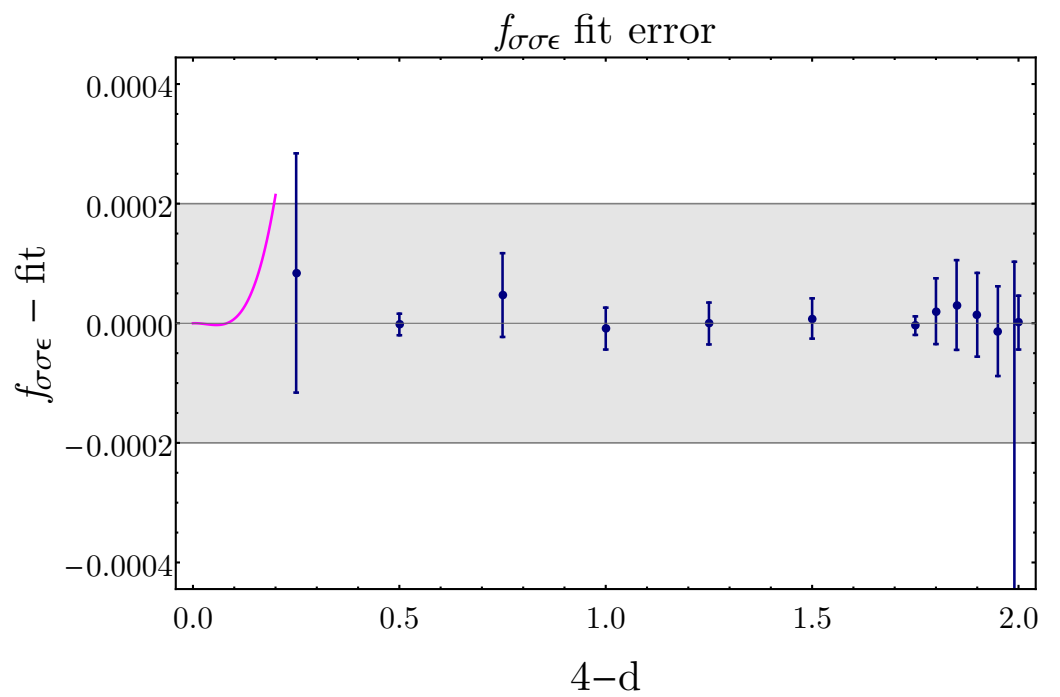

Figure 10. Fit of structure constant $f_{\sigma \sigma \epsilon}$ with bootstrap data (blue) with overall error (gray) and epsilon expansion (magenta) [31, 32].

The other four structure constants are determined with lower but yet good precision. Their polynomial fits are:

$$
\begin{aligned}
f_{\sigma \sigma \epsilon^{\prime}}(y)= & 0.130874 y-0.0916396 y^{2}+0.0125655 y^{3}+0.00124417 y^{4} \\
f_{\sigma \sigma T^{\prime}}(y)= & 0.0268204 y-0.0170251 y^{2}-0.00048868 y^{3}+0.00126381 y^{4} \\
f_{\sigma \sigma C}(y)= & 0.1690309-0.121369 y+0.0166922 y^{2}+0.0027202 y^{3} \\
f_{\sigma \sigma C^{\prime}}(y)= & 0.0103034 y-0.0268178 y^{2}+0.0391145 y^{3}-0.0315244 y^{4} \\
& +0.0132141 y^{5}-0.00220012 y^{6}
\end{aligned}
$$




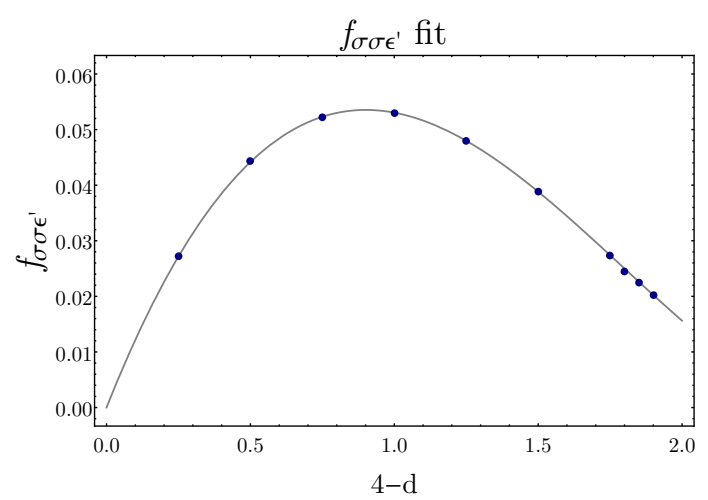

(a)

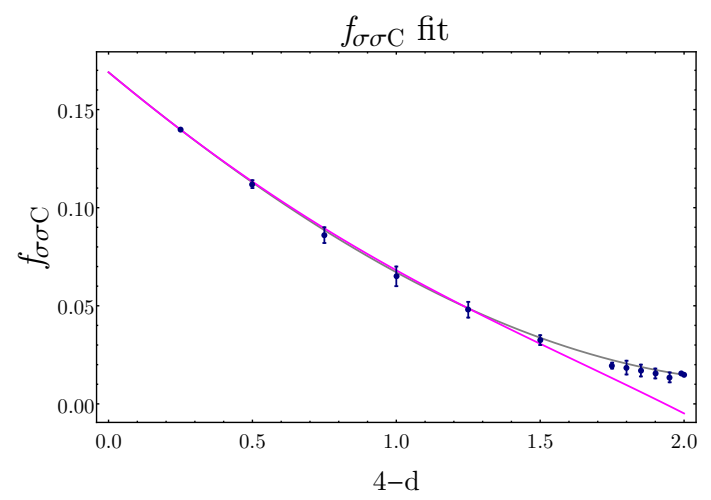

(c)

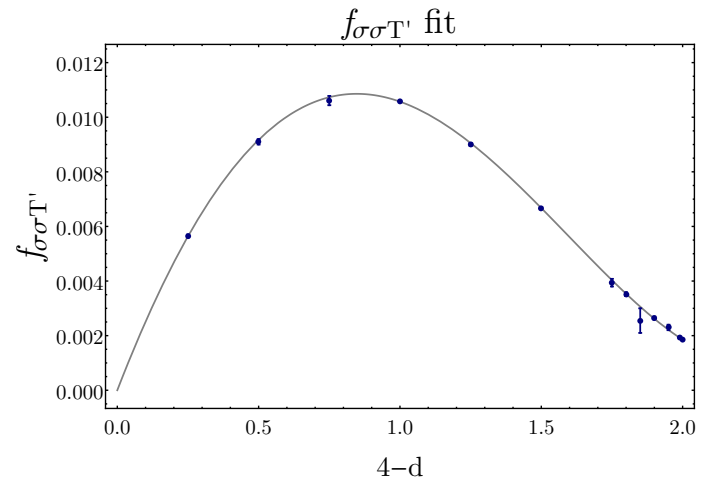

(b)

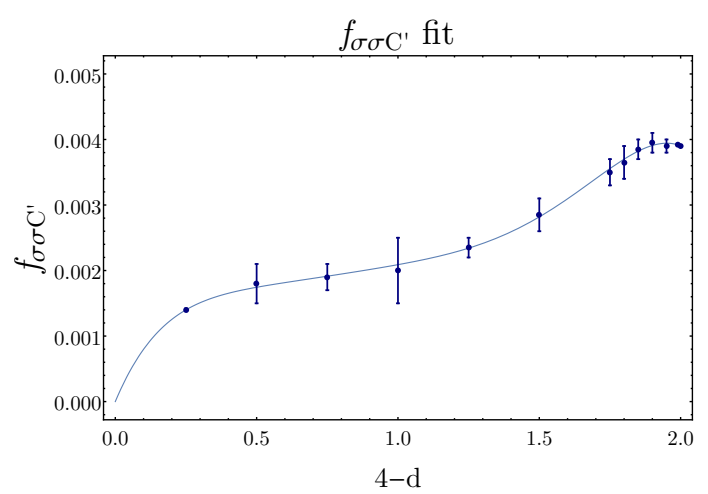

(d)

Figure 11. Polynomial fit of $f_{\sigma \sigma \epsilon^{\prime}}, f_{\sigma \sigma T^{\prime}}, f_{\sigma \sigma C}, f_{\sigma \sigma C^{\prime}}$, as functions of $y=4-d$. Figure (c) includes the known epsilon expansion result [31, 32].

and the corresponding errors are:

\begin{tabular}{|c|c|c|c|}
\hline & $4>d>2.2$ & $2.2 \geq d \geq 2.1$ & $2.1>d>\geq 2$ \\
\hline $\operatorname{Err}\left(f_{\sigma \sigma \epsilon^{\prime}}\right)$ & $<0.003$ & $\sim 0.007$ & $<0.003$ \\
$\operatorname{Err}\left(f_{\sigma \sigma T^{\prime}}\right)$ & $<0.0003$ & $\sim 0.001$ & $<0.0003$ \\
\hline
\end{tabular}

$$
\operatorname{Err}\left(f_{\sigma \sigma C}\right)<0.007, \quad \operatorname{Err}\left(f_{\sigma \sigma \mathbb{C}^{\prime}}\right)<0.0005 .
$$

The epsilon expansion is available for the $\ell=4$ leading-twist field $C$ [31-34]:

$$
\begin{aligned}
f_{\sigma \sigma C}(y)= & 0.16903085-0.12244675 y+0.02131741 y^{2}+0.002168567 y^{3} \\
& -0.0019760553 y^{4}, \quad \text { (Epsilon expansion). }
\end{aligned}
$$

The comparison with the bootstrap results is surprisingly accurate, as shown in figure 11(c). The plots of the other three structure constants are also shown in this figure.

\subsection{Analysis of data and applications}

The numerical bootstrap results in various dimensions obtained in this work have been recast in the form of simple polynomials of the variable $y=4-d$, spanning the range 
$4 \geq d \geq 2$. In the most precise cases, namely for the dimensions $\Delta_{\sigma}, \Delta_{\epsilon}, \Delta_{\epsilon^{\prime}}$, central charge $c$ and structure constant $f_{\sigma \sigma \epsilon}$, the error of the fits is rather small and bounded by a $d$-independent value. This has been chosen to be larger than the uncertainty of individual data points, so as to leave some space for potential unknown systematic errors of the bootstrap approach. All data show larger fluctuations in the region $2.3>d>2$, where the conformal theory rearranges itself to match the $d=2$ limit - a topic to be discussed in the next section.

The agreement with the existing results in the literature, often developed over decades of investigations, are very good: the bootstrap data, while being superior, are never inconsistent with them. In particular, the polynomials obtained here and those of the (unresummed) epsilon expansion are extremely close in their leading terms, allowing for precise matches in the region $4>d>3.8$.

The physical applications of these results are likely to be numerous: here, we shall mention two examples. The first one is the study of convergence for the epsilon expansion. This approach is straightforwardly and universally applied in field theory descriptions of critical phenomena, but it requires resummation techniques of the badly convergent perturbative series, such as the use of Borel transforms $[15,16]$. The precise data of our work can provide a clean case for checking and refining the optimization methods involved in the resummations $[17,21]$.

The second application is the study of the critical point of the Ising model with longrange interactions and its relation with the present, short-range case. This problem also has a long history and has been recently discussed in refs. $[19,20]$. In the definition of the model, the power-law decay of the spin-spin interaction $\alpha=d+s$ involves explicitly the space dimension and it is natural to discuss the equivalence/inequivalence of long-range and short-range universality classes by varying $d$ continuously. The precise data presented here can help testing the existing conjectures on the phase diagram [19, 20].

\subsubsection{The issue of unitarity}

In the recent work [37], the unitarity of the scalar field theory in non-integer dimension has been investigated. It is known that the free theory contains additional fields, called evanescent operators, whose correlators vanish algebraically at integer $d$, but involve negativenorm states for non-integer $d$. They occur for dimensions $\Delta \geq 15$ in $d=4$. After including the $\lambda \phi^{4}$ interaction, an example was found of an evanescent operator with $\Delta=23$ acquiring complex anomalous dimension to leading order in the epsilon expansion. These non-unitary states could be present in the critical Ising model for non-integer $d<4$.

Non-unitary states are problematic for the numerical bootstrap approach that assumes real $\Delta$ values and an expansion in conformal partial waves with positive coefficients $f_{\sigma \sigma \mathcal{O}}^{2}$. This issue has already been discussed in refs. $[8,37]$, leading to the conclusion that if nonunitary states appear in the partial-wave expansion of the four-spin correlator, they should be very high up in the spectrum and give negligible exponentially small contributions. The result of the three-correlator conformal bootstrap approach [8] at fractional dimensions $d=3.75,3.5,3.25$ is particularly interesting in this context, because it includes a larger set of partial waves and thus can better test non-unitarity for non-integer dimension. For these 
fractional $d$ values, it was found that the unitarity region shrinks to a small island in the $\left(\Delta_{\sigma}, \Delta_{\epsilon}\right)$ plane around the Ising point, but does not vanish within the precision attained.

In our work, we do not find instabilities of the numerical routines that could be interpreted as signs of non-unitarity, while reaching a precision that is superior to that of the mentioned works $[7,8]$. As a matter of fact, we must conclude again that the evanescent operators do not contribute significantly to the partial-wave expansion of the correlator $\langle\sigma \sigma \sigma \sigma\rangle$. We also note the good agreement for $4>d>3.8$ with the epsilon expansion results that do not rely on the unitarity of the theory. Let us finally quote the bootstrap approach of ref. [38] that does not require unitarity of the theory.

\section{Leading twists and the $d=2$ limit}

In this section we describe the conformal fields with lowest dimension for each spin value. In conformal theories with $d>2$, their anomalous dimensions $\gamma_{\ell}$, defined by $\Delta_{\ell}=d-2+\ell+\gamma_{\ell}$, are small numbers, with asymptotic large- $\ell$ value:

$$
\lim _{\ell \rightarrow \infty} \gamma_{\ell}=2 \gamma_{\sigma}
$$

Moreover, as a function of $\ell$, the $\gamma_{\ell}$ are fitted by a monotonically increasing and convex curve (Nachtmann theorem) [22-24]. Our analysis of the numerical bootstrap data will follow the same strategy of the previous section: first compare the $d=3$ data with the better three-correlator bootstrap results [13]; then, if this check is passed, extend the analysis to other $d$ values.

The data are again expressed by curves $\gamma_{\ell}\left(\Delta_{\sigma}\right)$ that fluctuate within the range of $\Delta_{\sigma}$ identified as the Ising theory, $0.51814 \leq \Delta_{\sigma} \leq 0.51817$ (see figure 1). Since this interval correspond to four data takes, we obtain four values of $\gamma_{\ell}$ for each $\ell$ value, that are represented as points in figure 12 (a) for $\ell=2,4, \ldots, 22$. In this figure, they are compared with the precise results of the three-correlator bootstrap (joined by the blue curve) and the asymptotic value (3.1) (grey line). One sees that the bootstrap solution oscillates between vanishing anomalous dimensions $\gamma_{\ell}=0$, corresponding to higher-spin conserved currents (free-theory), and non-vanishing values $\gamma_{\ell} \neq 0$. The presence of spurious freetheory solutions is a feature of the Extremal Functional Method already observed in [13].

We remark that the non-vanishing $\gamma_{\ell}$ values match rather well the blue curve for most spin values, namely the expected behavior is reproduced. Regarding the errors, in the previous section it was given by the fluctuation among the four data points; however, in the present case, this would be as large as the data value, corresponding to no predictions at all. Therefore, we shall consider an alternative point of view: we discard the free-theory $\gamma_{\ell}=0$ solutions and keep the non-vanishing ones, to which we do not assign a definite error. This choice is justified for the qualitative analyses carried out in this section.

It is also interesting to study the higher levels, i.e. the subleading twists, $\gamma_{\ell, 2}>\gamma_{\ell, 1} \equiv$ $\gamma_{\ell}$ : their values span a large range up to $\gamma_{\ell, 2} \sim 2.5$ for $\ell=22$ and show wide fluctuations. Nonetheless, some of the points lie slightly above the blue curve, as shown in figure 11(b): by inspection, one find that these $\gamma_{\ell, 2}$ values occur in combination with trivial first solutions $\gamma_{\ell}=0$ and thus are other would-be measures of leading-twist dimensions. However, 


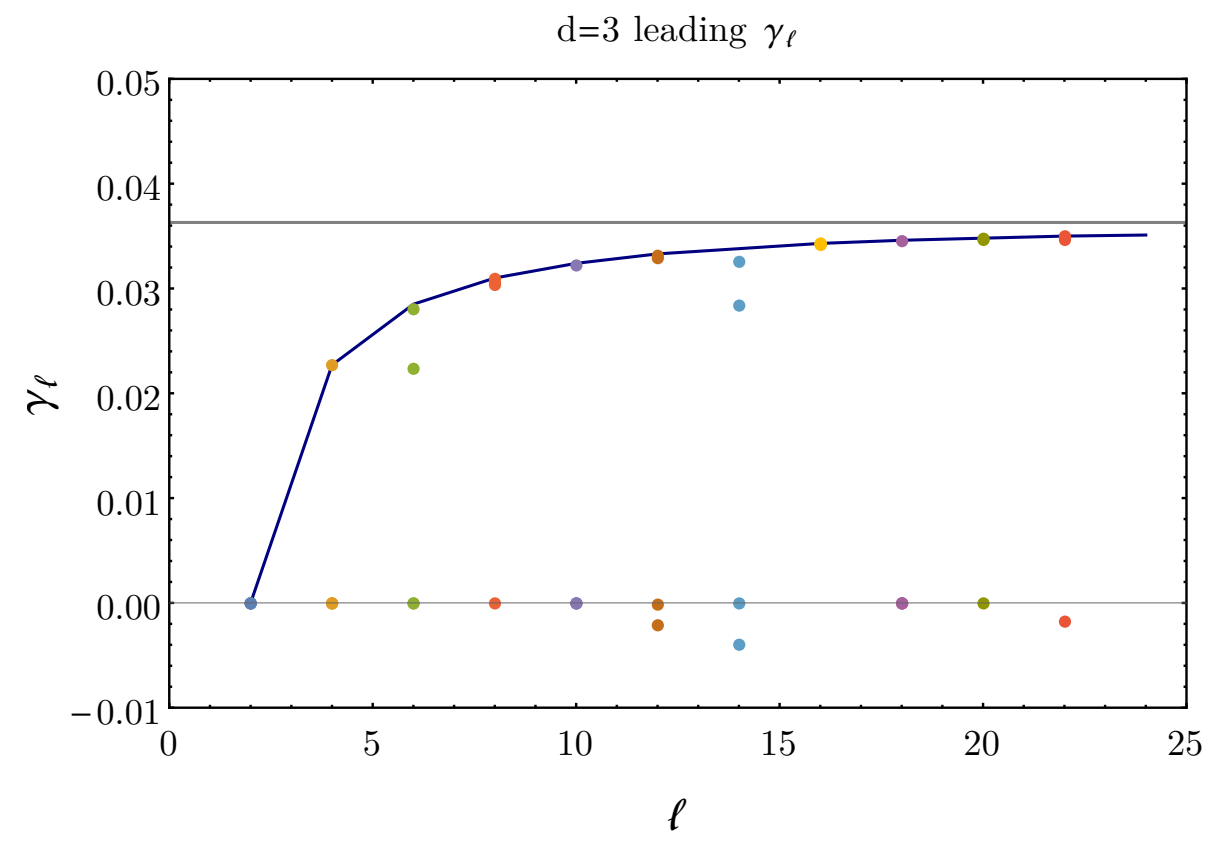

(a)

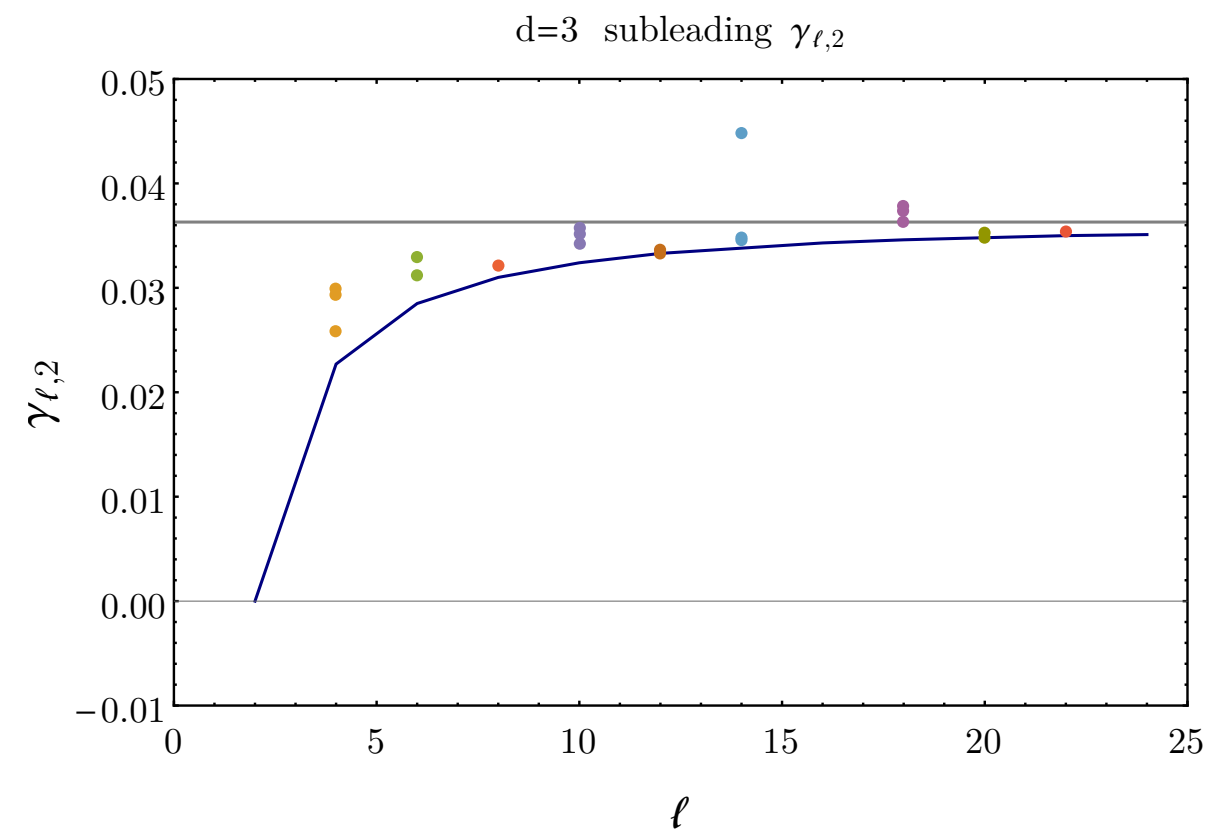

(b)

Figure 12. Anomalous dimension of leading (a) and subleading (b) twists with $2 \leq \ell \leq 22$ in $d=3$ compared with three-correlator results (blue curve) and $\ell \rightarrow \infty$ asymptotic value (gray line). 
their values are slightly overestimated, because they are pushed up by the level repulsion with $\gamma_{\ell}=0$.

In conclusion, the comparison with the precise three-correlator results [13] shows that the expected behavior at $d=3$ is well reproduced in our data by the leading states for any $\ell=2,4, \ldots, 22$, once the trivial cases $\gamma_{\ell}=0$ are discarded.

We now consider the results for other dimensions $3 \geq d \geq 2$. In figure 13(a), 13(b), we plot the $d$-dependence of the $\gamma_{\ell}(d)$ data, represented as points with colors associated to spin values, divided in two sets corresponding to $\ell=4 k$ and $\ell=4 k+2, k=1,2, \ldots$, respectively. Looking at the first set, we see that the $\ell$ sequences reach the asymptotic values $2 \gamma_{\sigma}(d)$ (blue curve) fairly well for $d=3,2.75,2.5$. However, starting from $d=2.25$, their behavior is different and the $\gamma_{\ell}$ gradually go to zero towards $d=2$, while approximatively keeping their monotonicity in $\ell$, i.e. $\gamma_{\ell}(d)<\gamma_{\ell+4}(d)$. In the case of the $\ell=4$ field $C$, this $d$-dependence was already present in figure 8 (c), but we now see that it holds for all $\ell$ values. At $d=2$, the leading twists become higher-spin conserved currents belonging to the Virasoro conformal block of the Identity field, as expected. The behavior of the second set of $\ell$ values in figure 13(b) is analogous, but there are stronger fluctuations and the monotonicity in $\ell$ is not always respected.

The observed $d$-dependence of the leading-twist dimensions $\gamma_{\ell}$ shows that the asymptotic limit (3.1) is not reproduced by our data for $d \leq 2.2$. Assuming a smooth transition to the $d=2$ spectrum, we may say that the asymptotic behavior becomes far fetched for $2.2>d>2$ and therefore the $\ell \rightarrow \infty$ and $d \rightarrow 2$ limits do not commute. Moreover, near $d=2$ the Nachtmann theorem becomes void. We conclude that at $d=2.2$ the towers of conformal fields begin to rearrange themselves to comply with the Virasoro representations at $d=2$.

The rearrangement of states is also visible for the subleading twists $\gamma_{\ell, 2}$, as shown in figure 14 for some selected $\ell$ values. The subleading states are above the blue curve, while the leading ones are below it, as in figure 13(a). We see that the $\gamma_{\ell, 2}$ anomalous dimensions are rather big for large $\ell$ values, but as $d$ is decreased they gradually converge to the common $d=2$ limit $\gamma_{\ell, 2}=1$ corresponding to the Virasoro conformal block of the energy field $\epsilon$. This behavior is clearer for the large $\ell$ values, since bigger $\gamma_{\ell, 2}$ values fluctuate less.

In conclusion, in this section we have shown that the leading-twist fields in our data match rather well the expected behavior (3.1) as a function of $\ell$ [22-24] for any dimension $3 \geq d>2.25$ and are also quantitatively good at $d=3$ (if trivial solutions $\gamma_{\ell}=0$ are disregarded). For $d \leq 2.25$, there is a change: the anomalous dimensions converge to zero in order to fit in the $d=2$ Virasoro conformal block of the identity field. The subleading twists similarly approach the conformal block of the energy. The transition region $2.25 \geq d>2$ is also characterized by the lower precision in the determination of conformal dimensions and structure constants as discussed in section 2.2. After the transition, the $d=2$ numerical data are again very good, even superior to the $d=3$ case.

This analysis yields a glimpse of the differences between conformal theories in two and higher dimensions we were alluding to in the Introduction. Although the low-lying states discussed in section 2 depend smoothly on dimension, the states higher up in the spectrum 


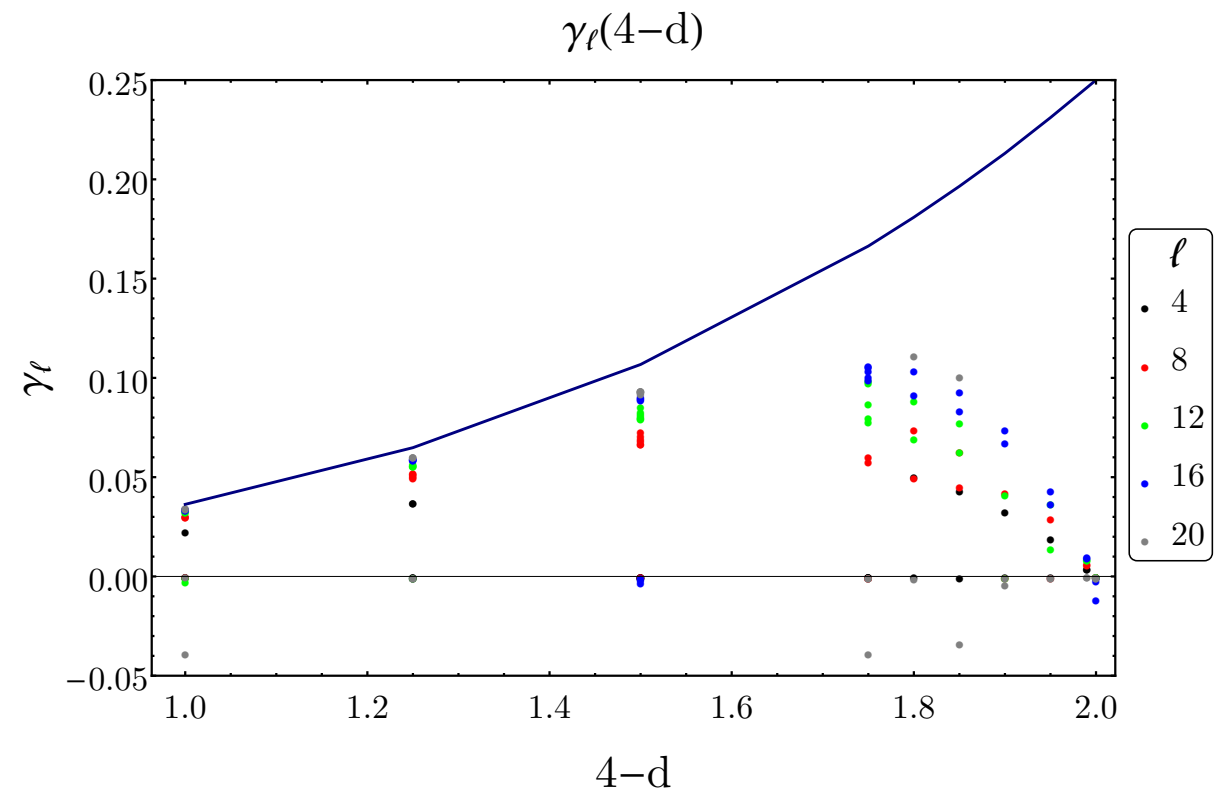

(a)

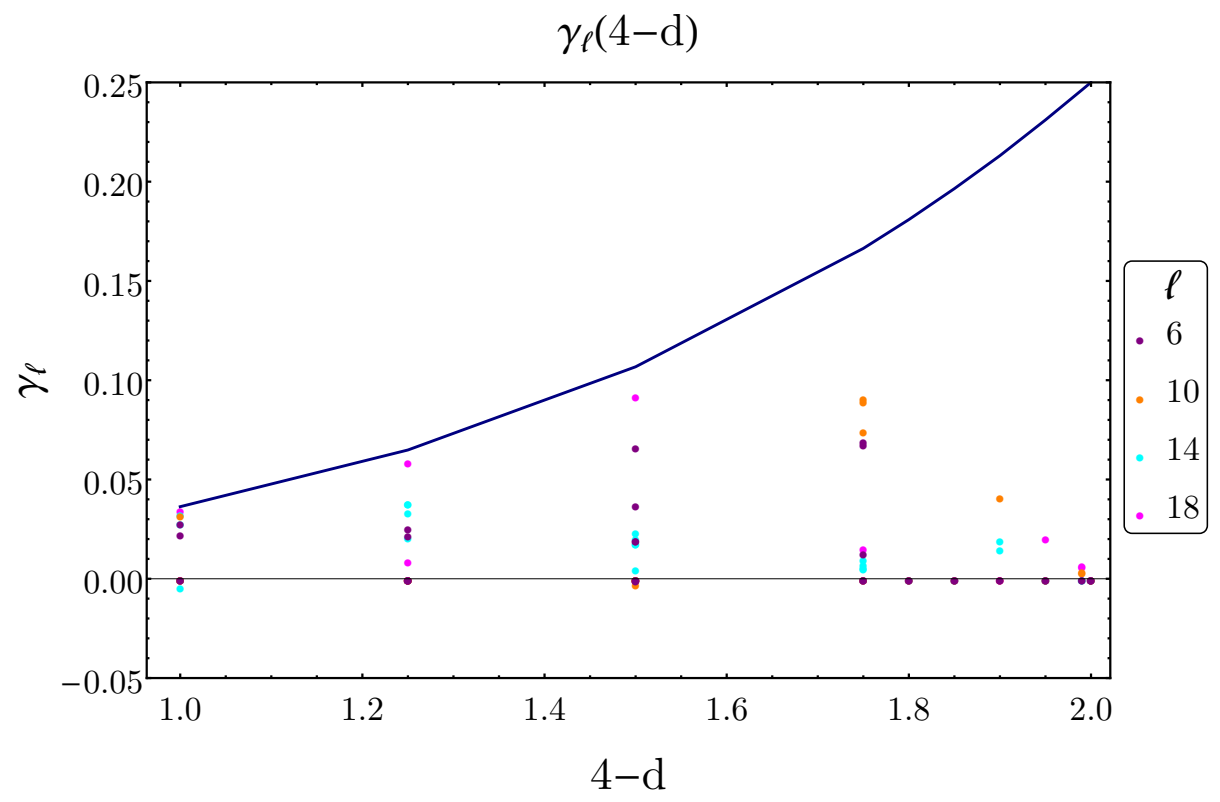

(b)

Figure 13. Dimension dependence of anomalous dimensions $\gamma_{\ell}(4-d)$ : for the $\ell$ values divided in two sets as given by the color tables. The blue curve shows the large $\ell$ asymptotic value equal to $2 \gamma_{\sigma}(4-d)$. 


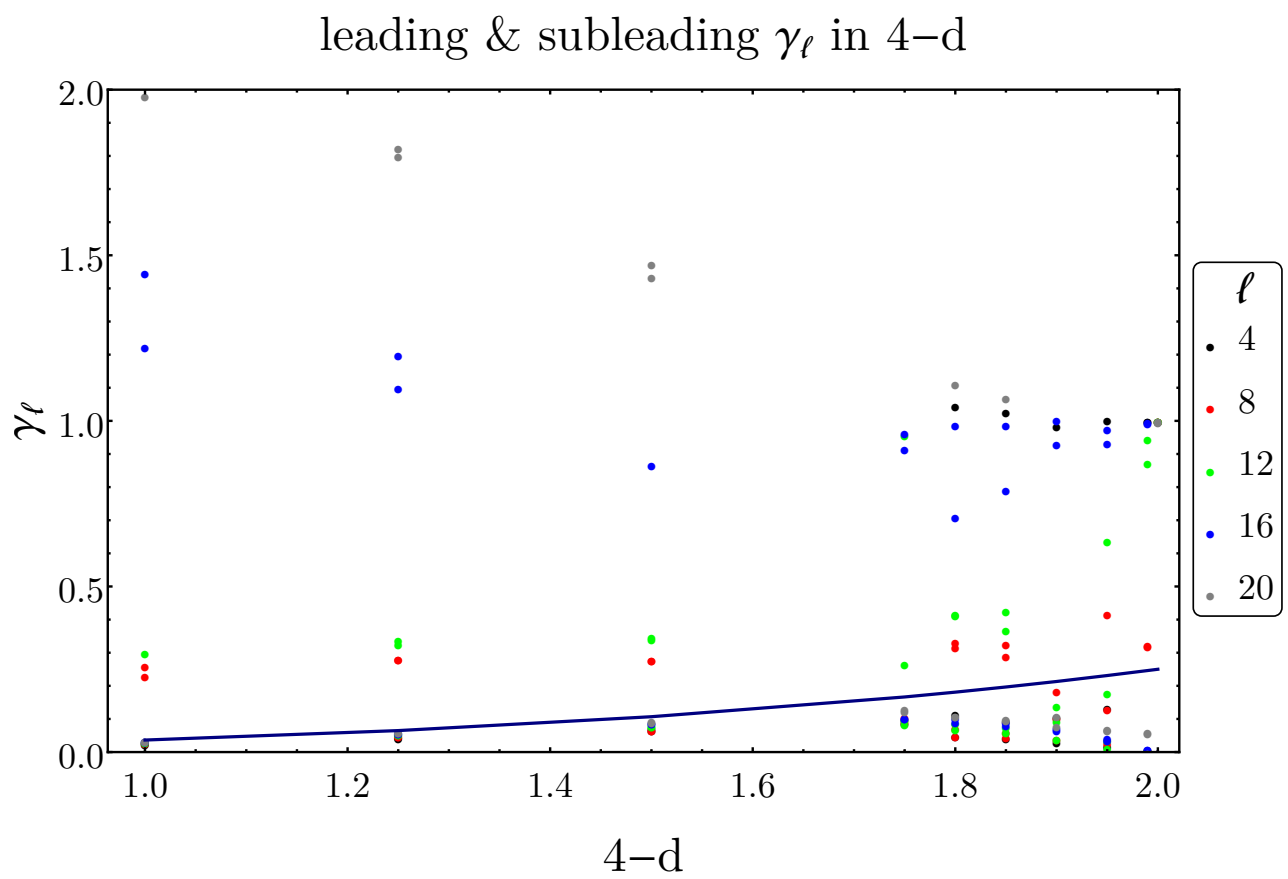

Figure 14. Dimension dependence of anomalous dimensions $\gamma_{\ell}(4-d)$ for the subleading twists (above the curve) for some $\ell$ values. The blue curve shows the large $\ell$ asymptotic value equal to $2 \gamma_{\sigma}(4-d)$.

change considerably and the Virasoro towers split up, with anomalous dimensions monotonically increasing with $\ell$. It would be interesting to study these phenomena analytically by developing an expansion in $d=2+\varepsilon$ (and possibly large $\ell$ ).

\section{State decouplings at the Ising point}

In this section we analyze the bootstrap data on the unitarity boundary in the vicinity of the Ising point and discuss the decoupling of states (vanishing structure constant) that take place while approaching this theory. In two dimensions, the Ising conformal bootstrap is obeyed by a highly reduced set of states, owing to the decoupling of Virasoro null (zeronorm) vectors. In higher dimensions, we generically expect a reduction of states for theories living on the unitarity boundary, where some squared structure constants parametrically change from positive to negative values. We should then search for specific decouplings as the Ising point is approached along the unitarity boundary, by tuning $\Delta_{\sigma}$. Our analysis will start by determining the structure constants that vanish in the $d=2$ model, then check those that are better identified numerically and follow their evolution for $2 \leq d \leq 3$.

This analysis is motivated by the observation that some structure constants for $\ell=0,2$ states do appear to vanish as approaching the $d=3$ Ising model [12]. In this Reference, the connection with the $d=2$ theory was already discussed: here we further analyze this issue using the high-quality data for $2 \leq d \leq 3$. 


\subsection{Counting of quasi-primary states on the $d=2$ unitarity boundary and Virasoro null vectors}

The conformal theory obeying the $d=2$ bootstrap equations on the unitarity boundary has been previously identified as the following non-unitary interpolation of the $c<1$ Virasoro minimal models. Their central charge and conformal dimensions are parameterized by the integer $m$ and read (Kac table) [2]:

$$
\begin{aligned}
c & =1-\frac{6}{m(m+1)}, \quad m=3,4, \ldots, \\
h_{r s} & =h_{m-r, m+1-s}=\frac{((m+1) r-m s)^{2}-1}{4 m(m+1)}, \quad 1 \leq r \leq m-1, \quad 1 \leq s \leq m .
\end{aligned}
$$

The conformal fields corresponding to spin and energy of the Ising model are identified as follows:

$$
\begin{aligned}
\sigma=\phi_{12}, & \Delta_{\sigma}=2 h_{12}=\frac{1}{2}-\frac{3}{2(m+1)}, & & \frac{1}{8} \leq \Delta_{\sigma} \leq \frac{1}{2}, \\
\epsilon=\phi_{13}, & \Delta_{\epsilon}=2 h_{13}=2-\frac{4}{m+1}, & & 1 \leq \Delta_{\epsilon} \leq 2 .
\end{aligned}
$$

The bootstrap equations for the $\langle\sigma \sigma \sigma \sigma\rangle$ correlator involve the conformal partial waves determined by the operator product expansion,

$$
\phi_{12} \cdot \phi_{12}=\phi_{11}+\phi_{13}
$$

that holds for any $m$ value, and is identified here as $\sigma \cdot \sigma=I+\epsilon$.

The interpolating conformal theory is obtained by assuming real $m \geq 3$ values. Eliminating this parameter from $\Delta_{\sigma}, \Delta_{\epsilon}$ in (4.2), one obtains the curve:

$$
\Delta_{\epsilon}=\frac{2}{3}\left(1+4 \Delta_{\sigma}\right)
$$

that matches the unitarity boundary found numerically at $d=2$ to the right of the Ising point, i.e of the kink in figure 3(c) [7]. Note also that the central charge, $c=\Delta_{\sigma}(5-$ $\left.4 \Delta_{\sigma}\right) /\left(1+\Delta_{\sigma}\right)$, correspondingly interpolates between the values $1 / 2 \leq c \leq 1$, i.e. over all minimal models. The inverse relation $\Delta_{\sigma}=[5-c-\sqrt{(c-1)(c-25)}] / 8$ identifies the field $h_{12}$ as one of the two Virasoro representations with null vector at level 2, the other being $h_{21}$. The next minimal model encountered on the unitarity line on the right of the Ising model is the Tricritical model for $m=4$ with $\Delta_{\sigma} \equiv 2 h_{12}=1 / 5$ and $\Delta_{\epsilon} \equiv 2 h_{13}=6 / 5$. Regarding the boundary on the left of the Ising point, the corresponding theories are believed not to correspond to any conformal theory since basic Virasoro descendants are missing in the spectrum [12].

In the following, we count the number of (quasi)-primary states that occur in the bootstrap partial waves, i.e. are generated by the OPE (4.3), in the cases of the Ising and Tricritical Ising models. From this counting we shall obtain the list of low-lying states that decouples as approaching the Ising model from the right, i.e. for $\Delta_{\sigma} \rightarrow(1 / 8)^{+}$, along the unitarity boundary, namely the states that are proper null vectors of this model. We shall adapt a counting argument due to A. Zamolodchikov [25]. 
The characters of Virasoro representations $\chi_{h}^{\mathrm{Vir}}(q)$ with weight $h$ and central charge $c$ are generating functions for the multiplicities $d_{N}(h, c)$ of descendant states, as follows [2]:

$$
\widetilde{\chi}_{h}(q)=\sum_{N=0}^{\infty} d_{N}(h, c) q^{N}=q^{-h+c / 24} \chi_{h}^{\operatorname{Vir}}(q)
$$

Among the Virasoro descendants, the quasi-primary fields cannot be written as derivatives of other fields: in terms of states, $|h, Q P\rangle \neq L_{-1} \mid h$, desc. $\rangle$. The corresponding multiplicities $d_{N}^{Q P}$ are then given by $d_{N}^{Q P}=d_{N}-d_{N-1}$ (with $d_{-1}=0$ ). It follows that the generating function for quasi-primary states is given by:

$$
\widehat{\chi}_{h}(q)=\sum_{N=0}^{\infty} d_{N}^{Q P} q^{N}=(1-q) \tilde{\chi}_{h}(q) .
$$

In the case of the Identity representation, one should take into account that the vacuum already obeys $L_{-1}|0\rangle=0$, thus the previous expression is modified as follows,

$$
\widehat{\chi}_{0}(q)=\sum_{N=0}^{\infty} d_{N}^{Q P} q^{N}=(1-q) \widetilde{\chi}_{0}(q)+q .
$$

For non-degenerate unitary representations of the Virasoro algebra with $c \geq 1$, the number of descendants at level $N$ is equal to the number of partitions of $N$ [2]; one readily finds the following generating functions:

$$
\widehat{\chi}_{h}(q)=\left(\prod_{k=2}^{\infty}\left(1-q^{k}\right)\right)^{-1}, \quad \widehat{\chi}_{0}(q)=(1-q)\left(\prod_{k=2}^{\infty}\left(1-q^{k}\right)\right)^{-1}+q .
$$

In the case of the critical and tricritical Ising models, one should insert the known form of the Virasoro characters for the representation $(r, s)$ of the $m$-th minimal model [2] into eq. (4.5), (4.6). The result is:

$$
\widehat{\chi}_{r s}(q)=\left(\prod_{k=2}^{\infty}\left(1-q^{k}\right)\right)^{-1} \sum_{n=-\infty}^{\infty} q^{n^{2} m(m+1)}\left(q^{n((m+1) r-m s)}-q^{r s+n((m+1) r+m s)}\right) .
$$

This formula is modified by adding $(+q)$ for $r=s=1$ and is going to be used for the values $m=3,4$.

In order to obtain the generating function $\mathcal{N}$ of quasi-primary multiplicities entering the $\langle\sigma \sigma \sigma \sigma\rangle$ bootstrap, i.e. of conformal partial waves, we consider the Virasoro representations in the r.h.s. of the operator product expansion (4.3) and write the expression:

$$
\begin{aligned}
\mathcal{N}_{m}(q, \bar{q}) & =\left|\widehat{\chi}_{11}(q)\right|^{2}+\left|q^{h_{13}} \widehat{\chi}_{13}(q)\right|^{2} \\
& =\sum_{\Delta=0}^{\infty} \sum_{|\ell| \leq \Delta} d^{Q P}(\Delta, \ell) x^{\Delta} y^{\ell}, \quad q=x y, \quad \bar{q}=x y^{-1}
\end{aligned}
$$

In this formula, the generating sum is rewritten in terms of conformal dimensions $\Delta=$ $N+\bar{N}$ and spins $\ell=N-\bar{N}$ of descendant states. 
We now discuss the counting of quasi-primaries in the interpolating theory on the unitarity boundary between IM and TIM, i.e. for $1 / 8<\Delta_{\sigma}<1 / 5$. The formula $\mathcal{N}_{m}$ (4.10) with $m=4$ describes the TIM case and this counting remains valid for lower $\Delta_{\sigma}$ values approaching the IM from the right, only the value $h_{13}=\Delta_{\sigma} / 2$ changes. Such extrapolation of $\mathcal{N}_{4}$ to $\Delta_{\sigma}=1 / 8^{+}$should be compared with the expression $\mathcal{N}_{3}$ that holds at the IM point $\Delta_{\sigma}=1 / 8$.

The multiplicities $d^{Q P}(\Delta, \ell)$ obtained by these two formulas are given in table 6 for the low-lying states (only even $\ell$ values are relevant). The counting for a generic $c>1$ theory is also shown for comparison; this is obtained by using the expressions (4.8) for the characters in (4.10) (in all cases $h_{13}=1 / 2$ ).

The analysis of the three tables leads to the following observations:

- The generic theory contains many more quasi-primaries than any minimal model, thus confirming the reduction of states on the unitarity boundary. More importantly, the IM has less states than the TIM. The latter fact follows from the properties of Virasoro representations. Any primary field $\phi_{r s}$ has a first null vector at level $N=r s$, that projects out a sub-tower of states: in the case of the energy field $\epsilon=\phi_{13}$ appearing in the operator product expansion (4.3), this occurs at level $N=3$. At the IM point, the reflection symmetry of the $m=3$ Kac table (4.1) implies the following field identification:

$$
\phi_{13} \equiv \phi_{21} .
$$

Thus, an additional degeneracy occur at level $N=2$, implying a further projection of states. Other projections due to higher- $N$ null vectors are taken into account in the expressions of the characters (4.9).

- In the case of a given correlator, such as $\langle\sigma \sigma \sigma \sigma\rangle$, the $d^{Q P}(\Delta, \ell)$ quasi-primary states for a given $(\Delta, \ell)$ pair combine in a single amplitude and a unique structure constant. Some amplitudes could be vanishing for this particular observable, thus the counting argument gives an upper bound of the possible bootstrap channels, in general. Since the expression of the $\langle\sigma \sigma \sigma \sigma\rangle$ correlator is known exactly for the interpolating theory, the structure constants as a function of $\Delta_{\sigma}$ have been obtained in refs. [12, 36, 39]. By inspection, one finds that the channel $(\Delta, \ell)=(11,0)$ is absent (green digit in the TIM spectrum of table 6$)$, while all the other ones are present.

- The comparison of the TIM and IM tables shows the following interesting cases:

$$
\begin{aligned}
& d_{T I M}^{Q P}(\Delta, \ell) \geq d_{I M}^{Q P}(\Delta, \ell)>0, \\
& d_{T I M}^{Q P}(\Delta, \ell)>0 \text { and } d_{I M}^{Q P}(\Delta, \ell)=0 .
\end{aligned}
$$

While moving from the TIM to the IM on the unitarity boundary, the first case gives a channel that remains open in the limit to the IM and therefore the corresponding state/field $\mathcal{O}(\Delta, \ell)$ exists at $\Delta_{\sigma}=1 / 8$. In the second case, the channel is open in the TIM but should close reaching the IM, thus the following decoupling should be observed:

$$
\lim _{\Delta_{\sigma} \rightarrow\left(\frac{1}{8}\right)^{+}} f_{\sigma \sigma \mathcal{O}(\Delta, \ell)}\left(\Delta_{\sigma}\right)=0
$$



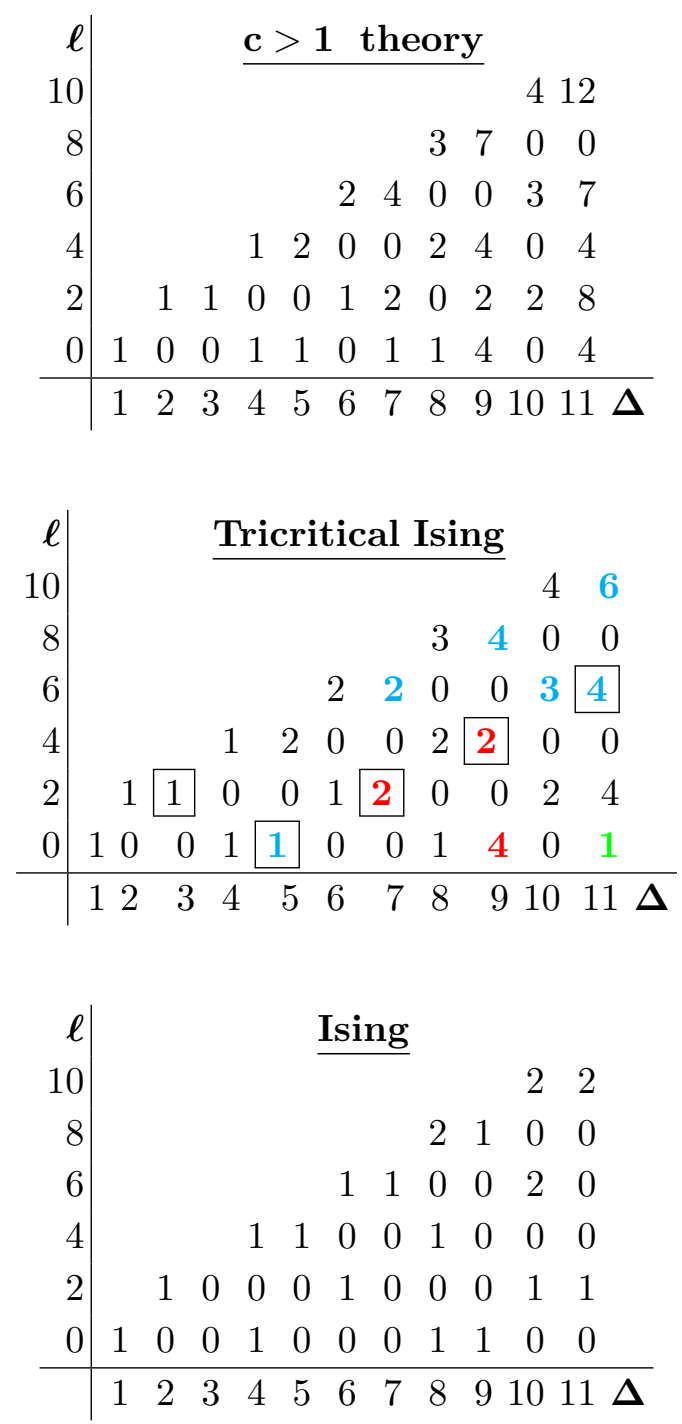

Table 6. Number of quasi-primary states in the $d=2\langle\sigma \sigma \sigma \sigma\rangle$ bootstrap as a function of conformal dimension and spin, for a generic $c>1$ theory, the tricritical and critical Ising Model, respectively. Boxed numbers indicate TIM channels that completely disappear in IM; cyan (red) numbers correspond to numerically uncertain (unseen) states; the green channel $(\Delta, \ell)=(11,0)$ does not appear in the four-spin correlator.

Therefore, the occurrence of pairs of multiplicities of the type (4.13), i.e. non-vanishing in TIM and vanishing in IM, gives a necessary condition for the decoupling of the corresponding state. Such occurrences within the low-lying spectrum are represented by boxed numbers in table 6 . The vanishing at $\Delta_{\sigma}=1 / 8$ of the corresponding structure constants has been checked by plotting the explicit expressions for $f_{\sigma \sigma \mathcal{O}}\left(\Delta_{\sigma}\right)$ obtained from the exact form of $\langle\sigma \sigma \sigma \sigma\rangle$. We stress that in the case of the state $(\Delta, \ell)=(11,0)$, the structure constant vanishes in the entire relevant region of $\Delta_{\sigma}$ and our argument based on (4.13) becomes void as well as irrelevant. 
In conclusion, the state decouplings discussed here could also have been obtained from the direct analysis of these structure constants, but we find that the counting argument is more instructive and general. Note also that all structure constants are real positive numbers in spite of the non-unitarity of the interpolating theory [37, 39].

\subsection{Numerical spectrum near the Ising point and decoupling of states for $2 \leq d \leq 3$}

The quality of the bootstrap data at $d=2.00001$ is also reported in table 6 using the following color code:

- Black digits represent states that are well observed and are close to the exact solution for $\Delta_{\sigma} \geq 1 / 8$.

- Red digits correspond to states not observed, owing to the large errors of subleading fields.

- Cyan digits represent observed states whose dimensions are not very close to the exact solution.

In conclusion, the decoupling of states at the Ising model can be described in our numerical setting for the well-seen spin-two state $(\Delta, \ell)=(3,2)$ and the less precise spin-zero state $(\Delta, \ell)=(5,0)$. These two decouplings are actually due to the $N=r s=2$ null vector of the energy field $\phi_{21}$ in the IM, eq. (4.11), in the chiral and scalar channels, respectively.

We now extend the analysis to $d>2$ by studying the behavior of the corresponding structure constants on the unitarity boundary to the right of the Ising points, $\Delta_{\sigma} \geq \Delta_{\sigma}^{*}$. The numerical results for the spin-two state are shown in figure 15. The vertical axis reports the logarithm of its structure constant $f_{\sigma \sigma \mathcal{O}(3,2)}$ as a function of $d$ and the displacement w.r.t. the Ising point $\Delta_{\sigma}-\Delta_{\sigma}^{*}$ (recall that this point is determined with error $<0.0001$ over the entire range). We find that the vanishing of the structure constant at $\Delta_{\sigma}=\Delta_{\sigma}^{*}$ continues above two dimensions, and smoothly connects to $d=3$. Therefore, the simplest level-two Virasoro null-vector condition at $d=2$ is found to correspond to the decoupling of the $\ell=2$ state observed earlier at $d=3$ [12].

This result gives remarkable support to the existence of specific state projections identifying the Ising conformal theory in three dimensions, one of the questions addressed in the Introduction. Let us postpone the discussion on $d>2$ null vectors to the Conclusions and analyze now the numerical precision of the result in figure 15.

In general, it is difficult to make precise quantitative comparisons of structure constants in the $d=2$ and $d=3$ theories, both in practice and in principle. Let us nonetheless report the numerical values for $f_{\sigma \sigma \mathcal{O}(3,2)}$ in the two cases, respectively at the critical point, the right edge of figure 15 and far off the Ising point:

\begin{tabular}{|c|ccc|c|}
\hline$\Delta_{\sigma}$ & $\Delta_{\sigma}^{*}$ & $\Delta_{\sigma}^{*}+0.002$ & $\Delta_{\sigma}^{*}+0.06$ & $\mathbf{d}$ \\
\hline$f_{\sigma \sigma \mathcal{O}(3,2)}$ & 0.0003 & 0.006 & 0.04 & $\mathbf{2}$ \\
$f_{\sigma \sigma \mathcal{O}(3,2)}$ & 0.003 & 0.03 & 0.2 & $\mathbf{3}$ \\
\hline
\end{tabular}




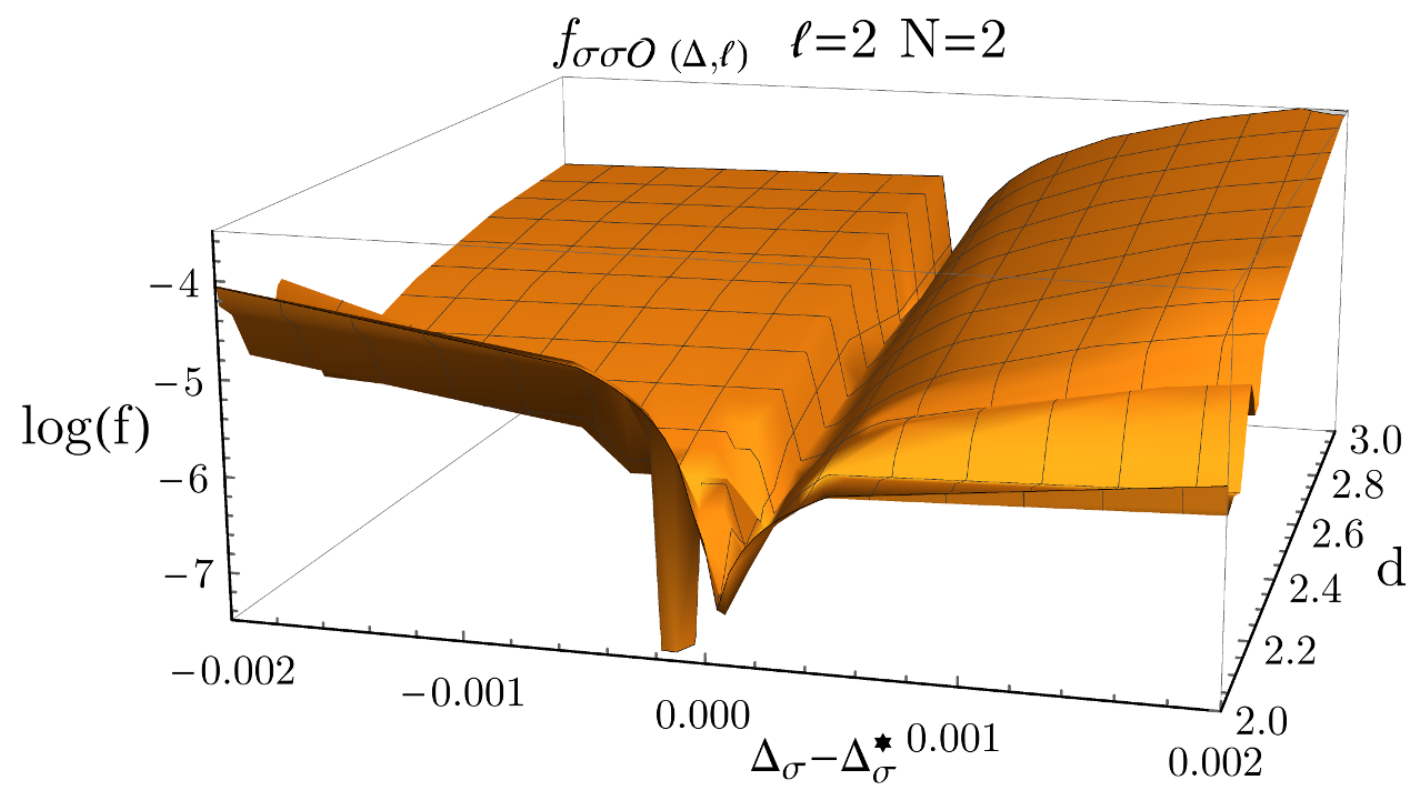

Figure 15. Evolution of the structure constant $f_{\sigma \sigma \mathcal{O}}$ for the $\ell=2$ null state as a function of dimension $d$ and displacement from the Ising point $\Delta=\Delta_{\sigma}^{*}$. The fluctuations observed to the right of $\Delta=\Delta_{\sigma}^{*}$ for $2 \leq d \leq 2.3$ are numerical uncertainties due to coarse sampling of the unitarity boundary. The big dip and the rise observed on the left of $\Delta=\Delta_{\sigma}^{*}$ for $d \geq 2$ are due to the decoupling of a left state that disappears at $d \sim 2.3$.

We see that the structure constant roughly drops one order of magnitude in the vicinity of the critical point and two orders w.r.t. its far-off value, both in the $d=2$ and $d=3$ cases.

Next, we give the values of other non-vanishing structure constants. In the case of the stress tensor, the leading $\ell=2$ state, $f_{\sigma \sigma T}$ is practically constant over the $\Delta_{\sigma}$ intervals, and reads:

$$
f_{\sigma \sigma T}=0.125(d=2), \quad 0.326(d=3) .
$$

These values are determined with $O\left(10^{-3}\right)$ relative error. The structure constant of the subleading field $T^{\prime}$, i.e. the next non-vanishing one in the $\ell=2$ channel, takes the following values at the Ising point $\Delta_{\sigma}^{*}$ :

$$
f_{\sigma \sigma T^{\prime}} \sim 0.002(d=2), \quad 0.01(d=3),
$$

and is determined with relative error $O\left(10^{-1}-10^{-3}\right)$.

The comparison of $f_{\sigma \sigma T}, f_{\sigma \sigma \mathcal{O}(3,2)}$ and $f_{\sigma \sigma T^{\prime}}$ for both dimensions shows that their offIsing values differ by roughly one order of magnitude relative to each other, i.e. follow the typical ordering of subleading states. However, at the Ising point the structure constant $f_{\sigma \sigma \mathcal{O}(3,2)}$ is one order of magnitude smaller than $f_{\sigma \sigma T^{\prime}}$ and comparable with the error of the latter. This behavior let us conclude that the minimal values taken by $f_{\sigma \sigma \mathcal{O}(3,2)}$ are consistent with zero within numerical precision.

The corresponding analysis of the structure constant for the $(\Delta, \ell)=(5,0)$ state shows that it goes to zero at the Ising point for $2 \leq d<2.2$, but stays constant above this 
dimension, namely this null state does not extend to $d=3$. The dimension at which the behavior changes is approximatively the same where the towers of leading-twist fields enter the higher-dimension regime, as discussed in sections three.

\section{Conclusions}

In this work, we have analyzed the critical properties of the Ising model in continuous dimension $4>d \geq 2$ by using the numerical conformal bootstrap. High quality data for the critical exponents and other low-lying conformal dimensions have been obtained together with the corresponding structure constants and have been fitted with simple polynomials in $d$. The comparison with other methods has shown good consistency and improvement.

Two qualitative results show the interplay between conformal field theories in $d=2$ and $d>2$. The first one is the behavior of leading and subleading-twist fields for spin $4 \leq \ell \leq 20$, that gradually acquire independent anomalous dimensions for $2<d<2.2$ and then enter the expected higher-dimensional regime for $d>2.2$. The second observation is the persistence above two dimensions of the decoupling of one state corresponding to the simplest $\ell=2$ null-vector of the Ising energy field $\epsilon=\phi_{21}$ in two dimensions. As discussed in the Introduction, this result is very intriguing and encouraging deeper analyses. For example, it would be interesting to develop analytic approaches based on $d=2+\varepsilon$ and/or large $\ell$ perturbative expansions [40].

Let us add some remarks concerning the possibility of specific state decouplings for the Ising model in $d>2$, i.e. of a consistent solution of the conformal bootstrap on a 'smaller' set of states. As emphasized in ref. [12], the presence of a kink, i.e. a singularity, on the unitarity boundary at the Ising point indicates that this model cannot be continuously deformed (even allowing some non-unitarity): this rigidity is already an indication of a smaller bootstrap. The main question is, in our opinion, how to formulate a consistent projection of states. Algebraic conditions directly involving critical exponents and other conformal data are not expected for $d>2$ - at least, a naive attempt to generalizing the level-two null-vector condition to $d>2$ has failed. Let us finally quote the following works analyzing the null-vectors of the $d>2$ conformal representations [41, 42].

\section{Acknowledgments}

The authors would like to thank D. Bernard, F. Gliozzi, R. Guida, Z. Komargodski, L. Rastelli, M. Serone and A. Trombettoni for interesting scientific exchanges, C. Behan and J. Henriksson for communicating their results [8, 33, 34], and in particular S. Rychkov for discussions, data [7] and comments on the manuscript. A. C. acknowledges the hospitality and support by the École Normale Supérieure, Paris, and all authors thank the G. Galilei Institute for Theoretical Physics, Arcetri, where part of this work has been done.

\section{A Numerical methods}

The conformal bootstrap for the four-point correlator $\left\langle\sigma\left(x_{1}\right) \sigma\left(x_{2}\right) \sigma\left(x_{3}\right) \sigma\left(x_{4}\right)\right\rangle$ of the scalar primary field $\sigma(x)$ with given conformal dimension $\Delta_{\sigma}$ is based on expanding the correlator 
in conformal partial waves (conformal blocks) and imposing the crossing symmetry. The resulting functional equation can be written:

$$
F_{0,0}(u, v)+\sum_{(\Delta, \ell) \neq(0,0)} p_{\Delta, \ell} F_{\Delta, \ell}(u, v)=0 .
$$

In this expression, the summation runs over the primary fields appearing in the $\sigma \cdot \sigma$ operator product expansion, with the exclusion of the identity field, whose contribution is singled out in $F_{0,0}(u, v) ; u$ and $v$ are the cross ratios,

$$
u=z \bar{z}=\frac{x_{12}^{2} x_{34}^{2}}{x_{13}^{2} x_{24}^{2}}, \quad v=(1-z)(1-\bar{z})=\frac{x_{14}^{2} x_{23}^{2}}{x_{13}^{2} x_{24}^{2}}
$$

the coefficients $p_{\Delta, \ell}=f_{\sigma \sigma \mathcal{O}(\Delta, \ell)}^{2}$ are squared structure constants and should be positive in an unitary theory. The functions $F_{\Delta, \ell}(u, v)$ are defined as:

$$
F_{\Delta, \ell}(u, v)=v^{\Delta_{\sigma}} G_{\Delta, \ell}(u, v)-u^{\Delta_{\sigma}} G_{\Delta, \ell}(v, u),
$$

where $G_{\Delta, \ell}(u, v)$ is a conformal block for the primary field with dimension $\Delta$ and spin $\ell$. The overall dependence on $\Delta_{\sigma}$ in all these formulas is left implicit.

In Reference [3], the problem of finding the spectrum of $(\Delta, \ell)$ and the coefficients $p_{\Delta, \ell}>0$ satisfying the function equation (A.1) and the unitarity constraint was reformulated into the following optimization problem. The functions $F_{\Delta, \ell}(u, v)$ (suitably discretized) are considered as elements of a vector space and a linear functional $\Lambda$ is introduced that should obey the following conditions:

$$
\begin{array}{rlrl}
\Lambda\left(F_{0,0}\right) & =1, & & \text { normalization, } \\
\Lambda\left(F_{\Delta, \ell}\right) \geq 0, & & \forall(\Delta, \ell) \text { in the spectrum. }
\end{array}
$$

If such a functional is found for a given spectrum $\{(\Delta, \ell)\}$, then the crossing-symmetry equation (A.1) cannot be satisfied for unitary theories and the corresponding conformal theory is ruled out.

The form of the functional over the truncated basis that we are going to use is:

$$
\begin{aligned}
\Lambda: F_{\Delta, \ell}(u, v) & \mapsto \sum_{0 \leq m+2 n \leq 2 n_{\max }+1} \lambda_{m, n} F^{(m, n)}(\Delta, \ell), \\
F^{(m, n)}(\Delta, \ell) & =\left.\partial_{a}^{m} \partial_{b}^{n} F_{\Delta, \ell}(a, b)\right|_{a=1, b=0},
\end{aligned}
$$

where $z=(a+\sqrt{b}) / 2$ and $\bar{z}=(a-\sqrt{b}) / 2$ and $\lambda_{m, n}$ are the coefficients defining the functional.

In our implementation, the calculation of the conformal blocks and their derivatives with respect to $a$ and $b$ follows the methods of ref. [43]. The conformal blocks along the diagonal $z=\bar{z}$ are expanded in series of $\rho=z /(1+\sqrt{1-z})^{2}$ :

$$
G_{\Delta, \ell}(z, z)=(4 \rho)^{\Delta} \sum_{n=0}^{\infty} b_{n} \rho^{n}, \quad b_{0}=1,
$$


where the $b_{n}$ are determined by recursion relations following from the quadratic and quartic Casimir equations. The series (A.6) is approximated by a polynomial with maximal degree $k_{\max }$, and the needed derivatives with respect to $a$ are easily obtained by using the map from $\rho$ to $a$. The derivatives with respect to $b$ are also determined by solving a recursion relation involving the quadratic Casimir (see appendix $\mathrm{C}$ of [26]).

Moreover, an additional approximation for the conformal blocks and their derivatives is introduced by replacing the poles at $\Delta_{j}$ with small residues $\theta_{j}<\theta$ with the sum of other poles with larger residues, as explained in appendices $\mathrm{A}$ and $\mathrm{B}$ of ref. [44]. The threshold values for the (normalized) residue are $\theta=10^{-8}$ for $3.75 \geq d \geq 2.01$ and $\theta=10^{-13}$ for $d=2.00001$. Note also that this approximation assumes that conformal blocks have simple poles, that is correct for dimensions $d \neq 2,4$. For this reason, the two-dimensional theory was studied at $d=2.00001$.

As a result, the functions $F^{(m, n)}(\Delta, \ell)($ A.5) are expressed as:

$$
F^{(m, n)}(\Delta, \ell) \sim \chi_{\ell}(\Delta) P_{\ell}^{(m, n)}(\Delta),
$$

where the factor $\chi_{\ell}(\Delta)$ is positive for all $\Delta$ in unitary theories and $P_{\ell}^{(m, n)}(\Delta)$ are polynomials. After removing the non-polynomial part $\chi_{\ell}(\Delta)$, the conditions (A.4) become:

$$
\begin{aligned}
& \sum_{0 \leq m+2 n \leq 2 n_{\max }+1} \lambda_{m, n} F^{(m, n)}(0,0)=1, \\
& \sum_{0 \leq m+2 n \leq 2 n_{\max }+1} \lambda_{m, n} P_{\ell}^{m, n}\left(\Delta_{\min }(\ell)+x\right) \geq 0, \quad x \in[0, \infty), \ell=0,2, \cdots, \ell_{\max },
\end{aligned}
$$

where $\Delta_{\min }(\ell)=D-2+\ell$ for $\ell>0$. Equation (A.8) is the final form that is used for implementing the numerical polynomial optimization by means of the SDPB solver [9]. These results are well established in the bootstrap literature and were summarized here for the sake of the presentation.

Next, the SDPB solver is run for various values of the dimension $\Delta_{\min }(0)$ of the lowest scalar primary field. Thus, we can identify the allowed and disallowed regions for the bootstrap, namely the unitarity boundary as a function of $\Delta_{\sigma}$ (see e.g. figure 1(a)). In our simulations, we used the functional with two values of $n_{\max }$ in (A.5), i.e. $n_{\max }=16$ and $n_{\max }=18$, corresponding to 153 and $190 F^{(n, m)}$ components, respectively. We also chose the maximal values $\ell_{\max }=50$ and $k_{\max }=120$ for all dimensions $d$. In table 7 we list the parameters of the SDPB solver that have been used in this work.

We now briefly summarize the implementation of the Extremal Functional Method [10, 11] for finding the spectrum of $(\Delta, \ell)$ values for states participating the bootstrap (A.1) and the corresponding structure constants $f_{\sigma \sigma \mathcal{O}(\Delta, \ell)}$. The functional (A.8) is called extremal when it is evaluated on the unitarity boundary, that has been identified in previous steps. The zeros of the extremal functional for each $\ell$ value identify the spectrum of dimensions $\Delta_{\ell, i}$ as functions of the boundary parameter $\Delta_{\sigma}$, that are the data discussed in the text.

The structure constants are obtained by solving the truncated crossing symmetry equations:

$$
F^{(m, n)}(0,0)+\sum_{(\Delta, \ell) \neq(0,0)} p_{\Delta, \ell} F^{(m, n)}(\Delta, \ell)=0
$$




\begin{tabular}{|l|l|}
\hline Parameter & Value \\
\hline findPrimalFeasible & true \\
findDualFeasible & true \\
detectPrimalFeasibleJump & false \\
detectDualFeasibleJump & false \\
precision & 704 \\
dualityGapThreshold & $10^{-30}$ \\
primalErrorThreshold & $10^{-30}$ \\
dualErrorThreshold & $10^{-30}$ \\
initialMatrixScalePrimal & $10^{20}$ \\
initialMatrixScaleDual & $10^{20}$ \\
feasibleCenteringParameter & 0.1 \\
infeasibleCenteringParameter & 0.3 \\
stepLengthReduction & 0.7 \\
choleskyStabilizeThreshold & $10^{-40}$ \\
maxComplementarity & $10^{100}$ \\
\hline
\end{tabular}

Table 7. Parameters employed in the SDBP program.

where the integers $(m, n)$ take all the positive values with $m+2 n \leq 2 n_{\max }+1$. The sums run over the $(\Delta, \ell)$ spectrum determined earlier. Since eq. (A.9) is an over-constrained linear system, the solution doesn't exist in general. Therefore, an approximated solution is found by considering the goal programming with the Chebyshev method [45, 46].

Open Access. This article is distributed under the terms of the Creative Commons Attribution License (CC-BY 4.0), which permits any use, distribution and reproduction in any medium, provided the original author(s) and source are credited.

\section{References}

[1] A.A. Belavin, A.M. Polyakov and A.B. Zamolodchikov, Infinite conformal symmetry in two-dimensional quantum field theory, Nucl. Phys. B 241 (1984) 333 [InSPIRE].

[2] P. Di Francesco, P. Mathieu and D. Senechal, Conformal field theory, Springer, Germany (1997).

[3] R. Rattazzi, V.S. Rychkov, E. Tonni and A. Vichi, Bounding scalar operator dimensions in $4 D$ CFT, JHEP 12 (2008) 031 [arXiv:0807.0004] [inSPIRE].

[4] D. Poland, S. Rychkov and A. Vichi, The conformal bootstrap: theory, numerical techniques and applications, Rev. Mod. Phys. 91 (2019) 015002 [arXiv:1805.04405] [INSPIRE].

[5] F.A. Dolan and H. Osborn, Conformal four point functions and the operator product expansion, Nucl. Phys. B 599 (2001) 459 [hep-th/0011040] [INSPIRE].

[6] F.A. Dolan and H. Osborn, Conformal partial waves and the operator product expansion, Nucl. Phys. B 678 (2004) 491 [hep-th/0309180] [InSPIRE].

[7] S. El-Showk et al., Conformal field theories in fractional dimensions, Phys. Rev. Lett. 112 (2014) 141601 [arXiv:1309.5089] [INSPIRE]. 
[8] C. Behan, PyCFTBoot: a flexible interface for the conformal bootstrap, Commun. Comput. Phys. 22 (2017) 1 [arXiv: 1602.02810] [INSPIRE].

[9] D. Simmons-Duffin, A semidefinite program solver for the conformal bootstrap, JHEP 06 (2015) 174 [arXiv: 1502 .02033] [INSPIRE].

[10] S. El-Showk and M.F. Paulos, Bootstrapping conformal field theories with the extremal functional method, Phys. Rev. Lett. 111 (2013) 241601 [arXiv:1211.2810] [INSPIRE].

[11] S. El-Showk and M.F. Paulos, Extremal bootstrapping: go with the flow, JHEP 03 (2018) 148 [arXiv: 1605.08087] [INSPIRE].

[12] S. El-Showk et al., Solving the 3d Ising model with the conformal bootstrap II. c-minimization and precise critical exponents, J. Stat. Phys. 157 (2014) 869 [arXiv:1403.4545] [INSPIRE].

[13] D. Simmons-Duffin, The lightcone bootstrap and the spectrum of the 3d Ising CFT, JHEP 03 (2017) 086 [arXiv: 1612.08471] [INSPIRE].

[14] F. Kos, D. Poland and D. Simmons-Duffin, Bootstrapping mixed correlators in the $3 D$ Ising model, JHEP 11 (2014) 109 [arXiv: 1406.4858] [INSPIRE].

[15] J.C. Le Guillou and J. Zinn-Justin, Accurate critical exponents for Ising like systems in noninteger dimensions, J. Phys. (Les Ulis) 48 (1987) 19.

[16] R. Guida and J. Zinn-Justin, Critical exponents of the N vector model, J. Phys. A 31 (1998) 8103 [cond-mat/9803240] [INSPIRE].

[17] M.V. Kompaniets and E. Panzer, Minimally subtracted six loop renormalization of $O(n)$-symmetric $\phi^{4}$ theory and critical exponents, Phys. Rev. D 96 (2017) 036016 [arXiv: 1705. 06483] [INSPIRE].

[18] M. Hasenbusch, Finite size scaling study of lattice models in the three-dimensional Ising universality class, Phys. Rev. B 82 (2010) 174433 [arXiv:1004.4486] [InSPIRE].

[19] C. Behan, L. Rastelli, S. Rychkov and B. Zan, A scaling theory for the long-range to short-range crossover and an infrared duality, J. Phys. A 50 (2017) 354002 [arXiv: 1703.05325] [INSPIRE].

[20] N. Defenu, A. Trombettoni and S. Ruffo, Criticality and phase diagram of quantum long-range $O(N)$ models, Phys. Rev. B 96 (2017) 104432 [arXiv:1704.00528] [INSPIRE].

[21] M. Serone, G. Spada and G. Villadoro, $\lambda \phi^{4}$ theory I: the symmetric phase beyond NNNNNNNNLO, JHEP 08 (2018) 148 [arXiv:1805.05882] [INSPIRE].

[22] O. Nachtmann, Positivity constraints for anomalous dimensions, Nucl. Phys. B 63 (1973) 237 [INSPIRE].

[23] Z. Komargodski and A. Zhiboedov, Convexity and liberation at large spin, JHEP 11 (2013) 140 [arXiv: 1212.4103] [INSPIRE].

[24] M.S. Costa, T. Hansen and J. Penedones, Bounds for OPE coefficients on the Regge trajectory, JHEP 10 (2017) 197 [arXiv: 1707.07689] [INSPIRE].

[25] A.B. Zamolodchikov, Integrable field theory from conformal field theory, in Integrable systems in quantum field theory and statistical mechanics, M. Jimbo et al. eds., Advanced Studies Pure Mathematics 19, Springer, Germany (1989).

[26] S. El-Showk et al., Solving the 3D Ising model with the conformal bootstrap, Phys. Rev. D 86 (2012) 025022 [arXiv:1203.6064] [INSPIRE].

[27] K.G. Wilson and M.E. Fisher, Critical exponents in 3.99 dimensions, Phys. Rev. Lett. 28 (1972) 240 [INSPIRE]. 
[28] A.M. Ferrenberg, J. Xu and D.P. Landau, Pushing the limits of Monte Carlo simulations for the three-dimensional Ising model, Phys. Rev. E 97 (2018) 043301 [arXiv:1806.03558] [INSPIRE].

[29] E. Vicari, Critical phenomena and renormalization-group flow of multi-parameter $\Phi^{4}$ field theories, PoS (LATTICE 2007) 023.

[30] A. Pelissetto and E. Vicari, Critical phenomena and renormalization group theory, Phys. Rept. 368 (2002) 549 [cond-mat/0012164] [INSPIRE].

[31] R. Gopakumar, A. Kaviraj, K. Sen and A. Sinha, Conformal bootstrap in Mellin space, Phys. Rev. Lett. 118 (2017) 081601 [arXiv: 1609.00572] [INSPIRE].

[32] R. Gopakumar, A. Kaviraj, K. Sen and A. Sinha, A Mellin space approach to the conformal bootstrap, JHEP 05 (2017) 027 [arXiv: 1611.08407] [INSPIRE].

[33] L.F. Alday, J. Henriksson and M. van Loon, Taming the $\epsilon$-expansion with large spin perturbation theory, JHEP 07 (2018) 131 [arXiv:1712.02314] [INSPIRE].

[34] J. Henriksson and M. Van Loon, Critical $O(N)$ model to order $\epsilon^{4}$ from analytic bootstrap, $J$. Phys. A 52 (2019) 025401 [arXiv:1801.03512] [INSPIRE].

[35] A.L. Fitzpatrick, J. Kaplan, D. Poland and D. Simmons-Duffin, The analytic bootstrap and AdS superhorizon locality, JHEP 12 (2013) 004 [arXiv:1212.3616] [INSPIRE].

[36] P. Liendo, L. Rastelli and B.C. van Rees, The bootstrap program for boundary $C F T_{d}$, JHEP 07 (2013) 113 [arXiv: 1210.4258] [INSPIRE].

[37] M. Hogervorst, S. Rychkov and B.C. van Rees, Unitarity violation at the Wilson-Fisher fixed point in 4- $\epsilon$ dimensions, Phys. Rev. D 93 (2016) 125025 [arXiv:1512.00013] [INSPIRE].

[38] F. Gliozzi and A. Rago, Critical exponents of the $3 d$ Ising and related models from conformal Bootstrap, JHEP 10 (2014) 042 [arXiv:1403.6003] [INSPIRE].

[39] C. Behan, Unitary subsector of generalized minimal models, Phys. Rev. D 97 (2018) 094020 [arXiv: 1712.06622] [INSPIRE].

[40] L.F. Alday, Large spin perturbation theory for conformal field theories, Phys. Rev. Lett. 119 (2017) 111601 [arXiv:1611.01500] [INSPIRE].

[41] S. Rychkov and Z.M. Tan, The $\epsilon$-expansion from conformal field theory, J. Phys. A 48 (2015) 29FT01.

[42] F. Gliozzi, A.L. Guerrieri, A.C. Petkou and C. Wen, The analytic structure of conformal blocks and the generalized Wilson-Fisher fixed points, JHEP 04 (2017) 056 [arXiv: 1702.03938] [INSPIRE].

[43] M. Hogervorst, H. Osborn and S. Rychkov, Diagonal limit for conformal blocks in d dimensions, JHEP 08 (2013) 014 [arXiv: 1305.1321] [INSPIRE].

[44] F. Kos, D. Poland and D. Simmons-Duffin, Bootstrapping the $O(N)$ vector models, JHEP 06 (2014) 091 [arXiv: 1307.6856] [INSPIRE].

[45] C. Romero, Handbook of critical issues in goal programming, Pergamon Press, New York U.S.A. (1991).

[46] D. Jones and M.Tamiz, Practical goal programming, Springer, Germany (2010). 